\title{
Multiphase Modelling of Pouring Glass over the Spout Lip of an Industrial Float in the Flat Glass Forming Process
}

\author{
Fernández Oro, J.M., Argüelles Díaz, K.M., Santolaria Morros, C \\ Universidad de Oviedo, Área de Mecánica de Fluidos. \\ Campus de Viesques, 33271, Gijón (Asturias), Spain. \\ jesusfo@uniovi.es \\ Cobo Hedilla, A.F., Lemaille, M. \\ Saint-Gobain Glass España, CIDA \\ Avda de Lugo 110, 33400 Avilés (Asturias), Spain \\ angel.cobo@saint-gobain.com
}

\begin{abstract}
This paper describes the numerical modelling of the three-phase flow that is established when pouring molten glass onto a tin bath (float) under a non-reactive atmosphere. Discharged from the furnace, the glass is spouted over a lip onto the bath, where it floats and spreads out in the form of a long ribbon, leading to the flat glass forming process. Numerically, the physical modelling must consider a multiphase problem with three inmiscible phases: glass, tin and air. The simulation of this complex free-surface flow involves the solution of the Navier-Stokes set of equations for all the phases simultaneously, using a volume-of-fluid (VOF) formulation that introduces a marker function convected by the flow to identify each phase. The evolution of the interphases is tracked over time with the implementacion of a continuous surface force (CSF) algorithm. A general purpose, welltested commercial code, FLUENT, is employed for the computations. Firstly, a twodimensional model considering the symmetry plane of the float is developed to fit accurate physical and numerical parameters. High complexity of the interphases in addition to great differences between the physical properties of the phases has required extensive tests to ensure the consistency and accuracy of the solutions. Afterwards, a complete threedimensional model is built to simulate the pouring process in a real geometry and predict the behaviour of the industrial facility when the operating conditions are modified. Typical flow phenomena inside the tin bath, like tin currents, wet back flow or even the equilibrium thickness, are also obtained as a promising result of the numerical modelling.
\end{abstract}

\section{INTRODUCTION}

The worldwide production of flat glass was approximately 42 million tonnes in 2006, with the perspective of a moderate growth in demand of 4 per cent per annum. Flat glass is basically consumed for windows in buildings ( $70 \%$ of the overall production), windshields for the automotive sector $(10 \%)$ and interior applications like TV screens and monitors $(20 \%)$. Basically, $90 \%$ of the world production of flat glass is manufactured using the flat glass forming process (float glass), developed by Pilkington [1] in 1959.

Float glass is produced by floating a continuous stream of molten glass delivered from the furnace onto a bath of molten tin (usually known as "float"). In order to achieve the proper thickness, the glass ribbon can be either pulled or stretched by toothed rollers at the edges of 
the sheet. As the glass flows down the bath, the temperature is gradually reduced allowing the solidification of the ribbon. Thus, when leaving the float, the sheet can be lifted from the tin onto rollers introducing it into an annealing lehr where is further cooled. The final result is a continuous supply of flat glass, with both sides being completely parallel and flat, which needs no additional manufacturing operations except cutting into plates for warehousing [2].

Definitively, the major advantage of the process is that glass, which is highly viscous, and the tin, which is very fluid, do not mix, so the contact surface between these two materials is perfectly flat. Moreover, the glass/tin system presents a perfect combination of both physical and chemical characteristics guaranteeing the success of the float process. In particular, both phases define an equilibrium thickness so the molten glass cannot be spread out indefinitely over the tin's surface. Then, if more glass is poured onto the bath, the ribbon enlarges horizontally at a constant thickness. This condition is a consequence of the balance between the influence of gravity and the effect of all the surface tensions involved at the three interfaces of the problem: glass/air, glass/tin and tin/air [3]. Hence, the equilibrium thickness is a characteristic property of the float process, which in the case of standard soda-lime glass under a protective atmosphere on a typical tin bath, is approximately $7 \mathrm{~mm}$. Therefore, the nature of the process leads to a glass thickness that is in the range of the typical industrial applications without needing external manipulation.

At present, high quality flat glass is increasingly demanded to satisfy the standards of value-added products in both automotive and electronic device sectors. In addition, the growth in demand for glass is leading to an increase in productivity ratios, either increasing the manufactured tonnes of glass or raising the production rate. Whatever the case, the float process is progressively overdemanded, leading to higher temperature and velocity gradients inside the tin bath. This negatively affects the stability of the glass sheet as well as the planimetry of the lower surface of the ribbon, inducing the formation of optical defects that cannot be corrected later [4]. Therefore, a deeper knowledge of the fluid dynamics of the process would lead to establishing accurate corrective actions and good practices when operating industrial floats under demanding conditions. Since the high temperatures inside the tin bath (up to $1100^{\circ} \mathrm{C}$ ) make it impossible to carry out detailed experimentation, analytical and numerical studies constitute the basic framework to characterize the float process.

Over the last 30 years, computational fluid dynamics (CFD) has become a powerful tool in predicting the performance of complex industrial processes. Over a wide variety of engineering applications, it is possible to perform simulations of complex problems, investigate the effect of different design parameters, obtain detailed distributions of all relevant variables, and gain insight into the underlying physical processes [5]. In the particular case of metallurgical flows, phenomena related to dynamics of interfaces and transport mechanisms are frequently observed as extremely complex situations [6]. Numerical studies can provide a comprehensive description of the industrial process thus avoiding timeconsuming and expensive experiments in engineering design. The use of CFD techniques has spread to different industrial situations and manufacturing processes, including mathematical modelling for flat glass production. In recent years, different approaches have been formulated to describe the float process [7]. However, the complexity of the fluid dynamics and heat transfer involved in the float glass process has led to simulate each phase of the problem separately. Some research efforts have been focused on the flow patterns inside the tin bath [4], while others have studied in detail the characteristics of the protective atmosphere [8]. The behaviour of the glass ribbon has been also of interest, especially when stretched by the top rollers $[9,10]$. Other numerical approaches have attempted coupling methods between the phases [11], or even the establishment of accurate numerical methodologies [12]. Nevertheless, most of the present numerical research related to glass forming is focused on the glass pressing process, like injection [13] and mould pressing by a plunger [14] or glass 
melting [15] using finite element techniques [16]. Nowadays, few relevant works regarding numerical simulation of the float process can be found in the literature, probably because of the commercial development of the float glass technology [3].

The mathematical modelling of interface dynamics, related to solidification and hot shaping of liquid metals, has been improved significantly over the last decade. In particular, the most established method employed in the modelling of metallurgical flows is the volumeof-fluid technique. In this period, a large number of phenomena have been studied numerically like metal transfer in arc welding [17], metal droplet splashing into a substrate [18] or filling of metals into moulds [19]. In the present paper, the volume-of-fluid method is used to perform a full 3D, three-phase model that simulates the pouring process of molten glass and how it spreads out under free conditions inside an industrial tin bath. With this approach, both volume-of-fluid and continuum surface force methods are revealed as a powerful numerical methodology to simulate the float glass process accurately. Firstly, a detailed analysis of all the relevant physical parameters is introduced in order to model properly the characteristics of the domain of interest. Then, several tests for consistency and accuracy are described, fixing the numerical parameters to provide good results. A twodimensional simulation is presented in the symmetrical plane of the float for that purpose. Third, the three-dimensional modelling of the process is achieved, analyzing the effect of different operating conditions over the glass ribbon profiles. Finally, typical flow phenomena inside the tin bath, like tin currents, wet back flow or even the equilibrium thickness, are also shown to highlight the potentiality and reliability of the numerical modelling.

\section{FLAT GLASS FORMING PROCESS. THE FLOAT PROCESS.}

In 1959, Pilkington Brothers Ltd. introduces an outstanding new method of glassmaking that revolutionized the glass industry in the last century (figure 1). In this process, a continuous ribbon of glass moves out of the melting furnace and floats along the surface of a bath of molten tin. There, the glass sheet is formed with the width and thickness required by the manufacturing needs. The valuable contribution of the float method consists in the continuous supply of flat glass with uniform thickness and bright, fire-polished surfaces without needing later grinding and/or polishing [20].

The raw materials (silica sand, calcium, oxide, soda and magnesium) are properly mixed together and then introduced into a furnace where they are melted at $1500^{\circ} \mathrm{C}$. Nowadays, modern furnaces can operate at up to 900 tonnes of glass per day, storing 2000 tonnes of melted material. The raw material, referred to as batch, blend together to form a large pool of an extremely viscous fluid, at very high temperatures, which flows out into the float through a delivery canal. This canal steadily narrows to end up with a subcritical spillway, usually known as spout lip (figure 2). After pouring out, the glass spreads outwards at the start of the float area, decreasing in thickness towards the equilibrium value. When the glass temperature is decreased to $900^{\circ} \mathrm{C}$, the glass viscosity is high enough to control the thickness of the glass ribbon using the top rolls. This operation pulls or pushes the edges of the glass sheet to decrease or increase the glass thickness respectively. This stretching process must conclude before the glass temperature reaches $800^{\circ} \mathrm{C}$. This process is readily calculated by equating the approximately constant tension to the Newtonian normal viscous stress and the pressure induced by these stresses [9]. From then on, the glass is conveyed freely, without strain, and cooled gradually to approximately $600^{\circ} \mathrm{C}$. With this temperature at the end of the float, the sheet can be lifted onto three rollers, travelling down into the annealing lehr [21]. Finally, the glass comes down to an open-air area where the final product is inspected and cut 
into plates. The tin bath is protected with a non-reactive atmosphere consisting of a mixture of nitrogen (approx. 94\%) and hydrogen (approx. 6\%) to prevent tin oxidation.

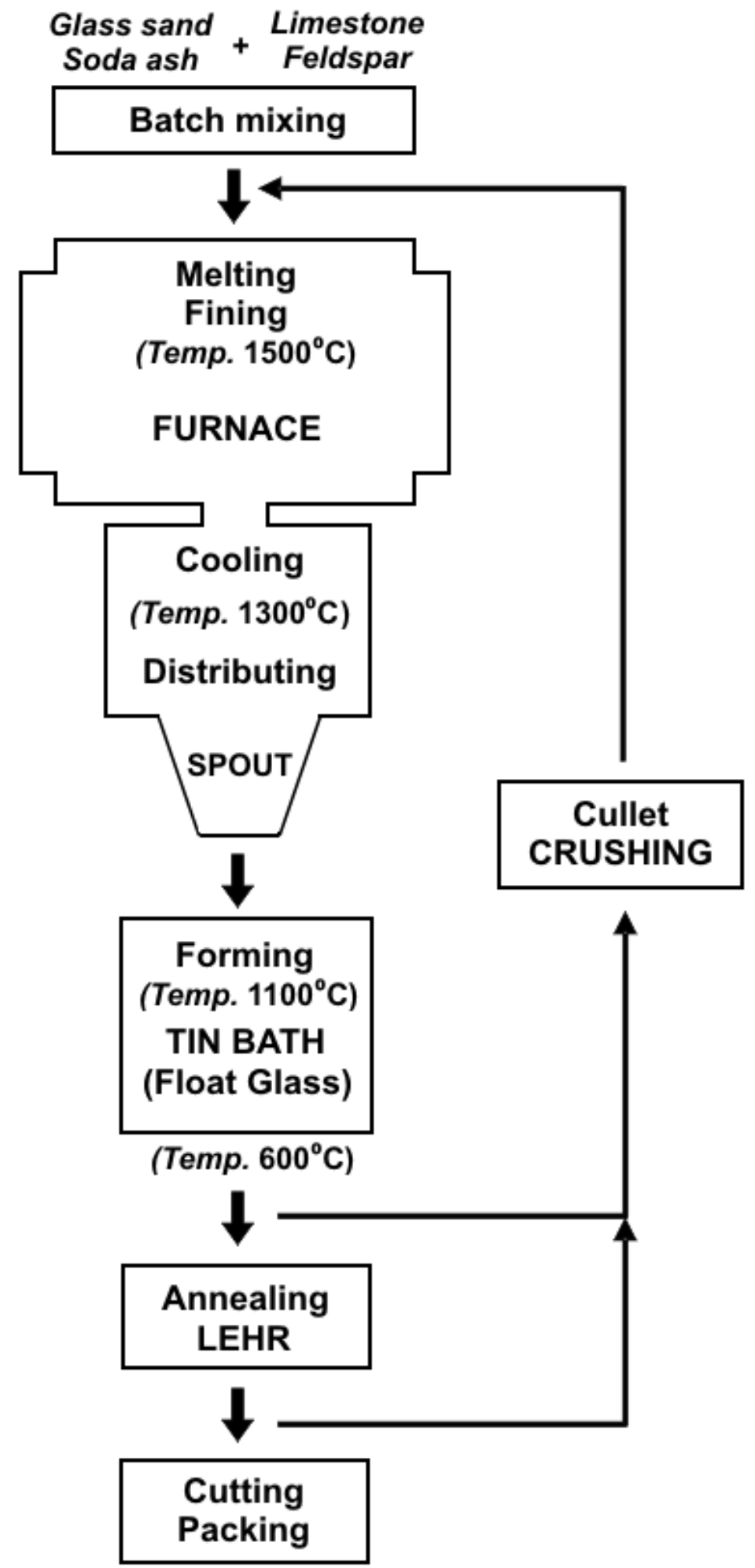

Fig. 1. Float process 


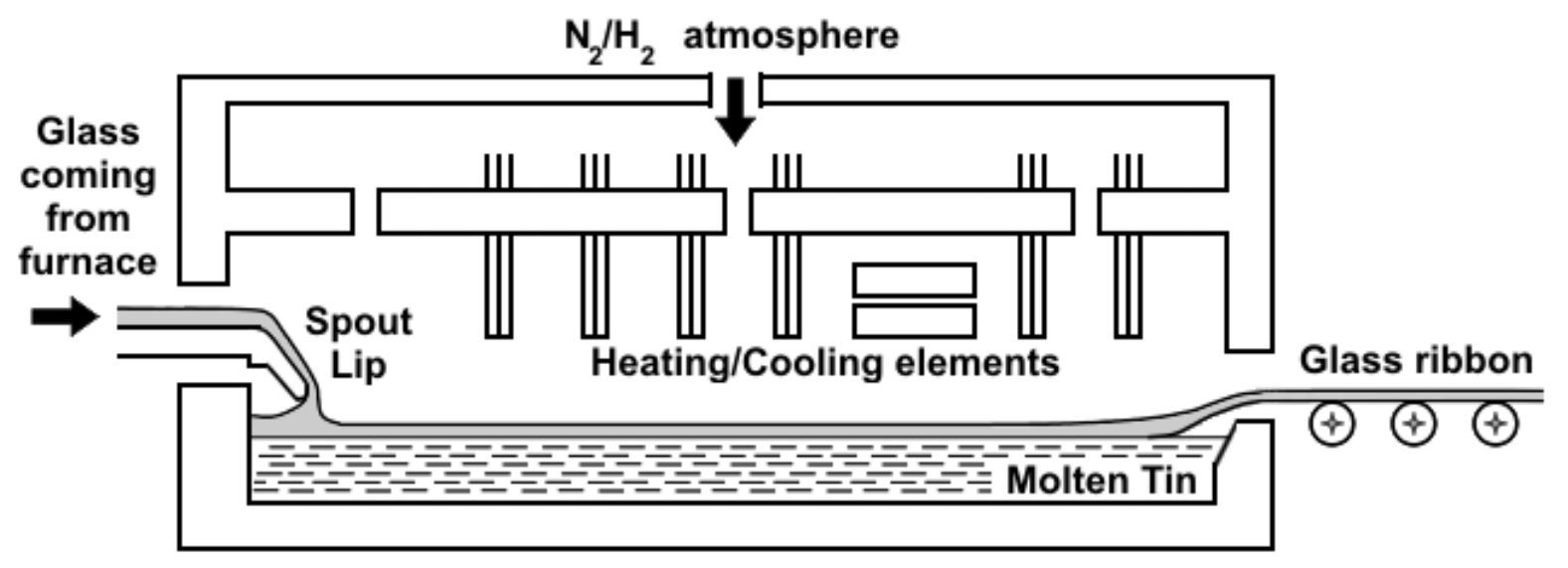

Fig. 2. The tin bath

Typical industrial floats are 60 meters long, 4 to 8 meters wide, and filled with molten tin to maximum levels that are between 4 and 10 centimetres. This results in 200 tonnes of molten tin. On the other hand, the glass suffers a gradual cooling from $1100^{\circ} \mathrm{C}$ to $600^{\circ} \mathrm{C}$, which implies a drastic change in both physical and rheological properties. The glass phase evolves from a highly-viscous liquid state to a plastic condition, practically solidified. This transformation is due to heat exchange transferred between the glass ribbon and the tin bath system. When the top rolls are working, important strain forces are applied on the glass sheet, exerted by several pairs of toothed rollers that control the glass thickness in the range between $1.1 \mathrm{~mm}$ and $20 \mathrm{~mm}$ (figure 3). Also, the float introduces water-cooled elements for the heat exchange and even heating elements (typically, electric resistances cantilevered from the bath roof) that are locally employed to adjust the glass cooling process when necessary.

Molten tin reacts with oxygen, particularly at elevated temperatures. The tin oxide thus formed appears as dross on the surface of the open tin and as deposits of tin oxide in the bath roof. This dross can result in glass defects, affecting product quality [22]. To minimize the oxidation, the protective atmosphere is introduced; however, no float bath is perfectly sealed and air ingress is an ongoing phenomenon. Thus, the unwanted dross is typically accumulated inside the dross boxes at the end of the float. These reservoirs must be periodically cleaned out.

The amount of glass allowed to pour onto the molten tin is controlled by a refractory gate called tweel (actually, it has a back-up device to prevent a breakdown). This flow control device, placed in the intermediate canal between the furnace and the float (figure 4), fixes the mass flow rate of the process. 


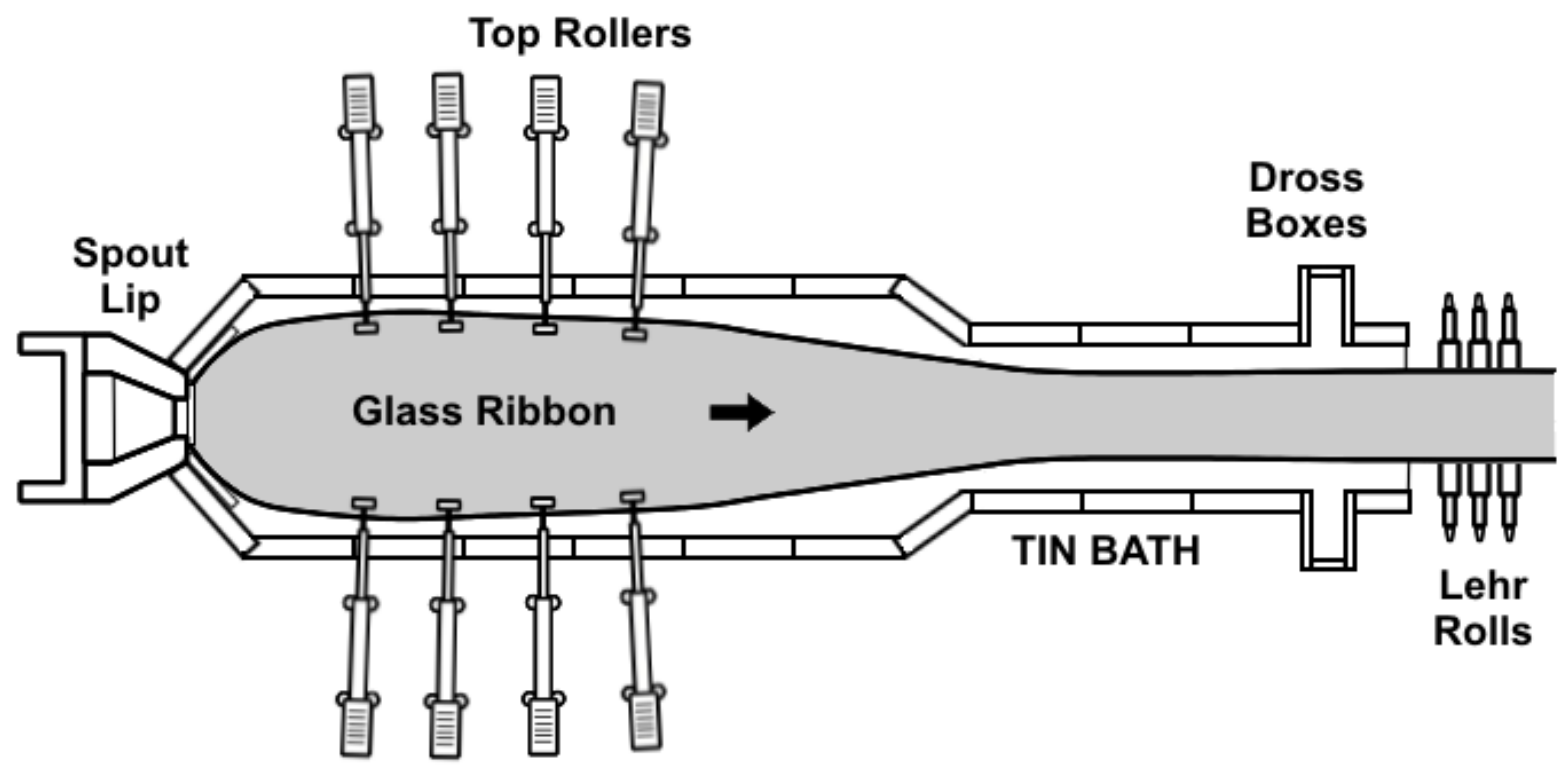

Fig. 3. Aerial view of a typical float

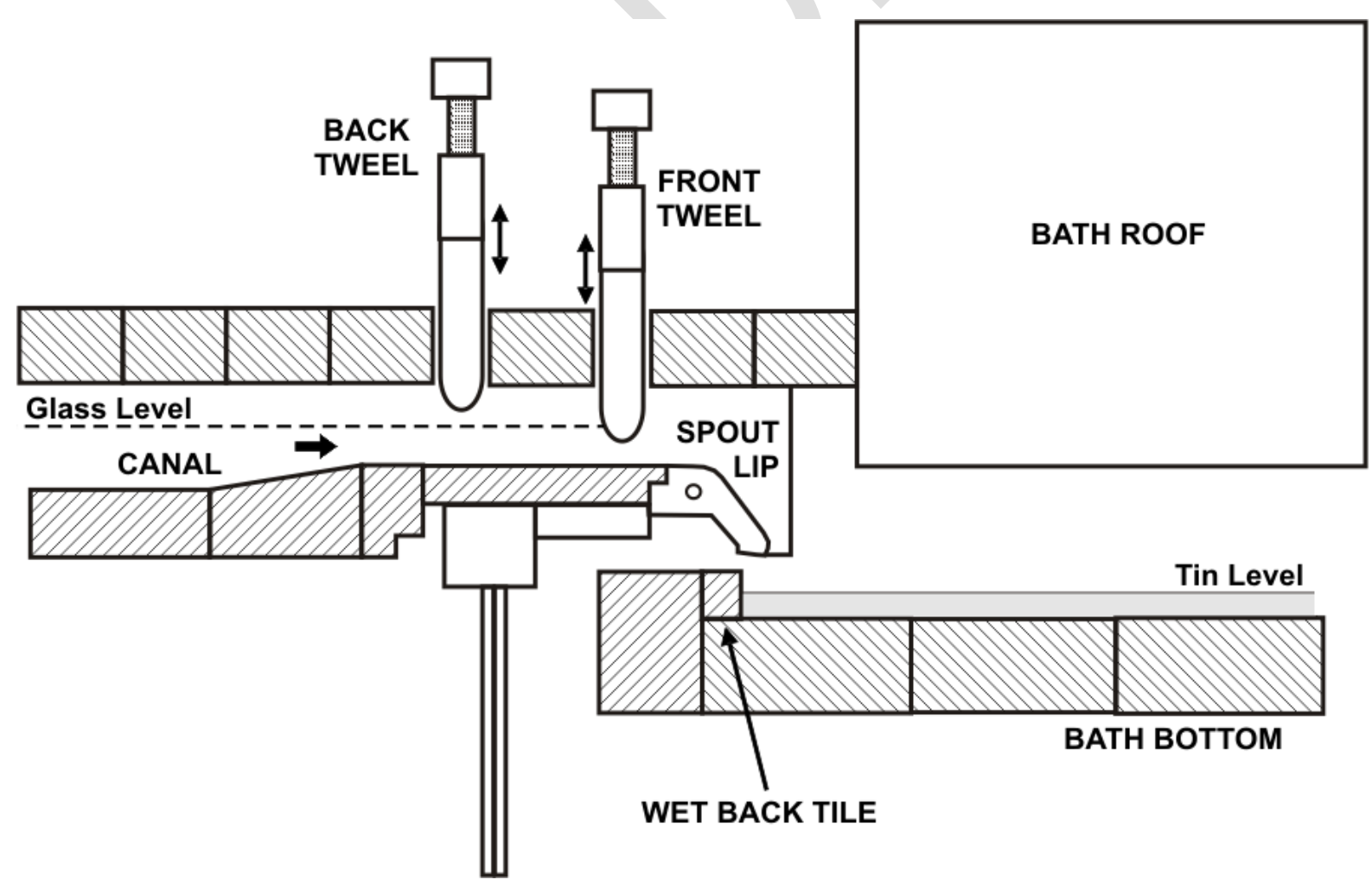

Fig. 4. Flow control device at the entrance area of the float. 


\section{TIN BATH FLUID DYNAMICS}

The physico-chemical phenomena involved inside the tin bath are so diverse and of such a complex nature that it is necessary to employ different scientific disciplines to analyze their global performance. For instance, the rheological evolution of the glass must be studied using material sciences, while the oxide formation has to be described by means of analytical chemistry. On the other hand, the analysis of the glass dynamics (while not vitrified) and the tin currents induced by the ribbon displacement raise questions to be answered by fluid dynamics.

The glass/tin system is fully adequate for the float process due to the perfect combination of both physical and chemical properties of the phases [12]. At first glance, all the compatibility conditions to avoid a chemical reaction between them over a wide range of temperatures are satisfied. In addition, the glass, which is not wet by the molten tin, presents a lighter density than the tin, approximately $2500 \mathrm{~kg} / \mathrm{m}^{3}$ vs. $6700 \mathrm{~kg} / \mathrm{m}^{3}$. Obviously, this is essential to allow the flotation of glass in a stable way. Also, the viscosity and the thermal conductivity of both phases present adequate behaviors. Particularly, the high glass viscosity varies enormously along the float (up to 10 orders of magnitude) because of its extreme dependence on temperature. On the contrary, the tin viscosity, slightly influenced by temperature, is always significantly lower than that of the glass, so the glass ribbon can always be considered a moving wall BC for the tin phase. Complementarily, the thermal conductivity of the tin is 40 times higher than that of the glass. Since the glass ribbon is the main source of heat of the float, the tin acts as a thermal diffuser, redistributing the temperature gradients and reducing the thermal stresses. Finally, the surface tensions also contribute to the characteristics of the system, providing an equilibrium thickness that has an effect on the glass sheet and matches perfectly within the range of typical industrial applications.

\section{Tin currents}

The tin dynamics constitute a perfect example of a confined flow in a fixed, closed volume. The depth of the tin is several orders lower than the other characteristic dimensions (width and length), so it is expected that the flow is severely stratified inside the bath. Once the equilibrium thickness is developed, the glass sheet can be considered as a solid contour for the tin phase. With this constraint, the tin motion is similar to that in a turbulent Couette flow [3]. Typical Reynolds numbers, based on the glass velocity and the tip depth, range from 20,000 to 100,000 in the case of industrial floats, the flow always being turbulent. Since the float contains around 200 tonnes of molten tin, this means that all the tin is completely recirculated in 20 minutes.

Initially, the basic function of the tin is to support the glass ribbon to allow the formation of the glass sheet. The great difference between the glass and tin densities (ratios range from $10^{6}$ to $10^{9}$ depending basically on the glass temperature) means that the glass behaves as a driving wall inducing important tin currents in the float. Note that these currents are also an important mechanism for the redistribution of the heat transfer. Therefore, two types of tin current can be distinguished inside the tin bath: a primary current, driven by the glass ribbon in the central zones of the float, and a secondary, recirculation current that moves upstream in the lateral zones of open tin [23].

Since these currents could be considerable, the stability of the glass sheet might be seriously compromised. In order to control these tin flows and minimize the impact of the global redistribution of heat, tungsten or graphite rods (tin barriers) are usually included to modify the hydrodynamics of the float. However, not only is the float a physical support for 
the glass ribbon, but also it can be considered as an equilibrium system with an ongoing thermal redistribution. The total heat power to be transferred is around $4 \mathrm{MW}$ when reducing the glass temperature to $500^{\circ} \mathrm{C}$. This large amount of heat must be evacuated by the cooling elements, by the surrounding atmosphere, but mainly by convective mechanisms in the tin bath. Therefore, the tin plays a dominant role in the thermal performance of the process [4].

\section{Flow patterns in the glass ribbon}

The dynamics of the glass ribbon are governed by the Navier-Stokes equations as long as the phase is still liquid. At the start of the float area, the elevated temperatures ensure the fluidity of the molten glass thus allowing the application of the fluid dynamics laws. Therefore, it is possible to analyze the pouring process over the spout lip and also the flotation mechanisms that produce the equilibrium thickness.

First of all, we consider briefly how the glass passes over the spout lip. In this case, the analysis is reduced to the classical problem of a thin film flowing down an inclined plane, assuming the following hypothesis: two-dimensional flow of a viscous, newtonian fluid [20] under laminar, steady-state conditions. Hence, the film thickness, $e_{l i p}$, can be estimated according to [24]:

$$
e_{l i p}=\left(\frac{3 \mu_{g}(Q / b)}{\rho_{g} g \cos \theta}\right)^{1 / 3}
$$

where $\rho_{g}$ and $\mu_{g}$ are the glass density and viscosity respectively, $g$ is the gravitational acceleration, $\theta$ is the inclination angle of the lip and $(Q / b)$ is the volumetric flow rate per unit width. Also, the velocity profile, $u$, of the thin film follows a parabolic law:

$$
u(y)=\frac{\rho_{g} g}{2 \mu_{g}} \cos \theta\left(2 e_{l i p} y-y^{2}\right)
$$

where $y$ represents the vertical coordinate, normal at the lip surface.

\section{Equilibrium thickness}

The equilibrium thickness is determined by considering the following assumptions: both glass and tin phases behave as viscous, newtonian fluids with constant physical properties. The glass flow is laminar, steady, fully-developed and two-dimensional. In addition, the transport process is taken to be adiabatic; because of the reduced dimensions of the domain of interest (the temperature field is barely reduced from inlet to outlet positions at the very start of the float).

The molten glass does not spread unimpeded over the surface of the molten tin. Despite the influence of gravity, it is restrained by surface tension acting in all the interphases: glass/air, $\sigma_{g}$, glass/tin, $\sigma_{g t}$ and tin/air, $\sigma_{t}$. The resulting equilibrium between the gravity and the surface tensions leads to the typical shape shown in figure 5 , defined by the equilibrium thickness of the molten glass, $\mathrm{T}$.

Vertically, the weight of the glass must be balanced by the buoyancy forces from the displaced tin. In the horizontal direction, the hydrostatic distributions are balanced by the 
surface tensions along the glass thickness, as represented in figure 5. Both effects are combined to give the following relation $[25,26]$ :

$$
T=\left(\frac{2 \rho_{t}\left(\sigma_{g}+\sigma_{g t}-\sigma_{t}\right)}{g \rho_{g}\left(\rho_{t}-\rho_{g}\right)}\right)^{1 / 2}
$$

This means that the equilibrium thickness depends exclusively on the surface tensions and densities of the phases. Substituting typical values, (see table 1), the equilibrium thickness is $6.75 \mathrm{~mm}$; i.e., approximately $7 \mathrm{~mm}$.

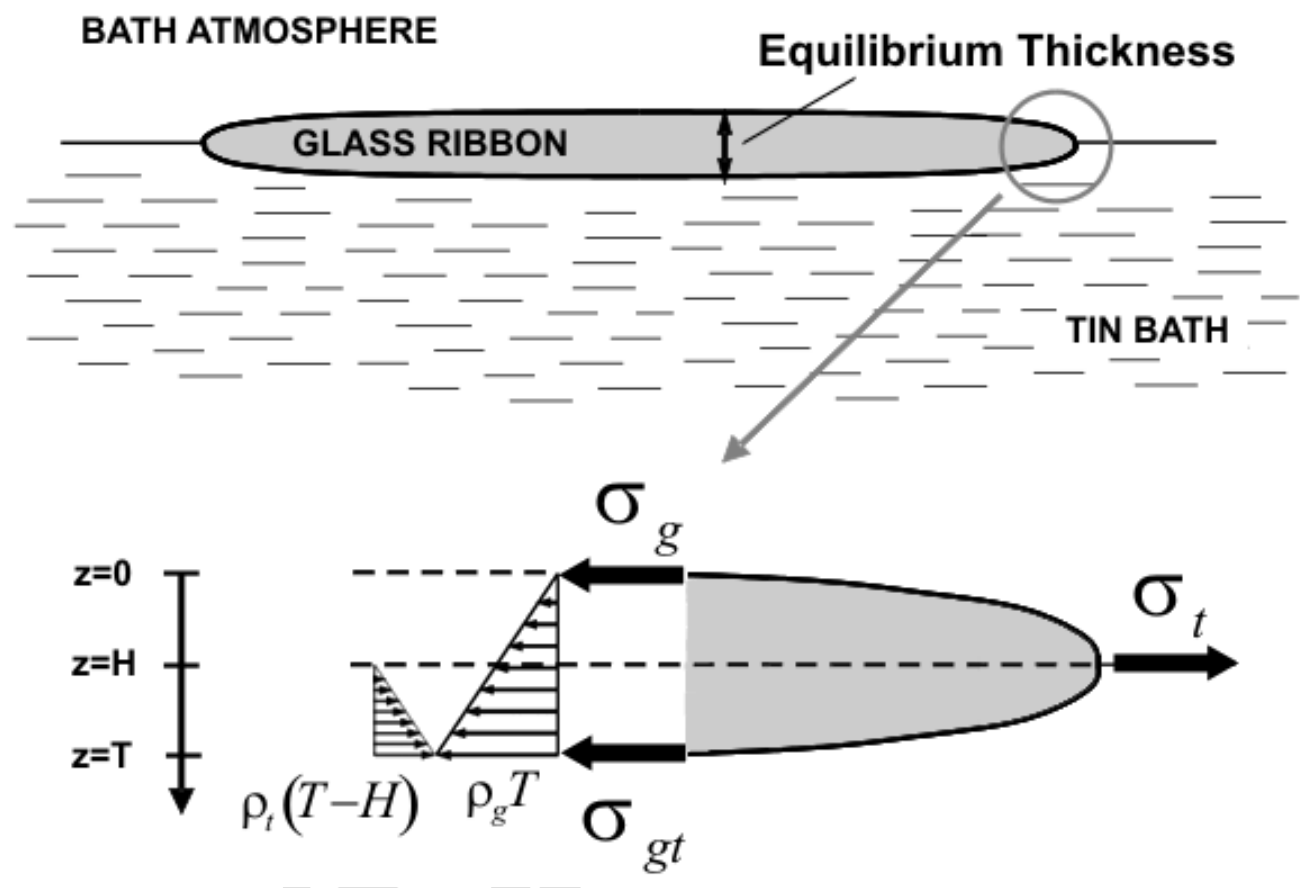

Fig. 5. Acting forces in the equilibrium thickness.

\section{"Wet Back Flow"}

The "Wet Back Flow" is a characteristic flow phenomenon of the float process, resulting from a backward flow of the glass poured. Basically, at the spot where the liquid glass meets the float bath, a shoulder is formed. Most of the glass flows forward in the direction of the float bath outlet, but a small amount flows backward. The part of the float tank in which the glass flows backward is referred to as the wetback region. The wetback region of the tank is more or less funnel-shaped, wider in the direction of the float tank outlet. The two sides of the "funnel" usually consist of ceramic tiles known as the restrictor tiles. The narrow part of the funnel is formed by the front wall of the float tank, a refractory tile placed over it, referred to as the wetback tile (WBT in figure 6).

The glass flowing backward touches the wetback tile and the restrictor tiles, is deviated by them and flows with the main part of the glass in the direction of the float tank outlet. The pool of glass appearing in the wetback region can cause defects in the glass. In the glass pool, the residence time of this glass on the float bath is longer than that of the glass 
flowing directly to the outlet. This can lead to a different viscosity, because the glass is cooled further, and even to devitrification and decomposition of the glass.

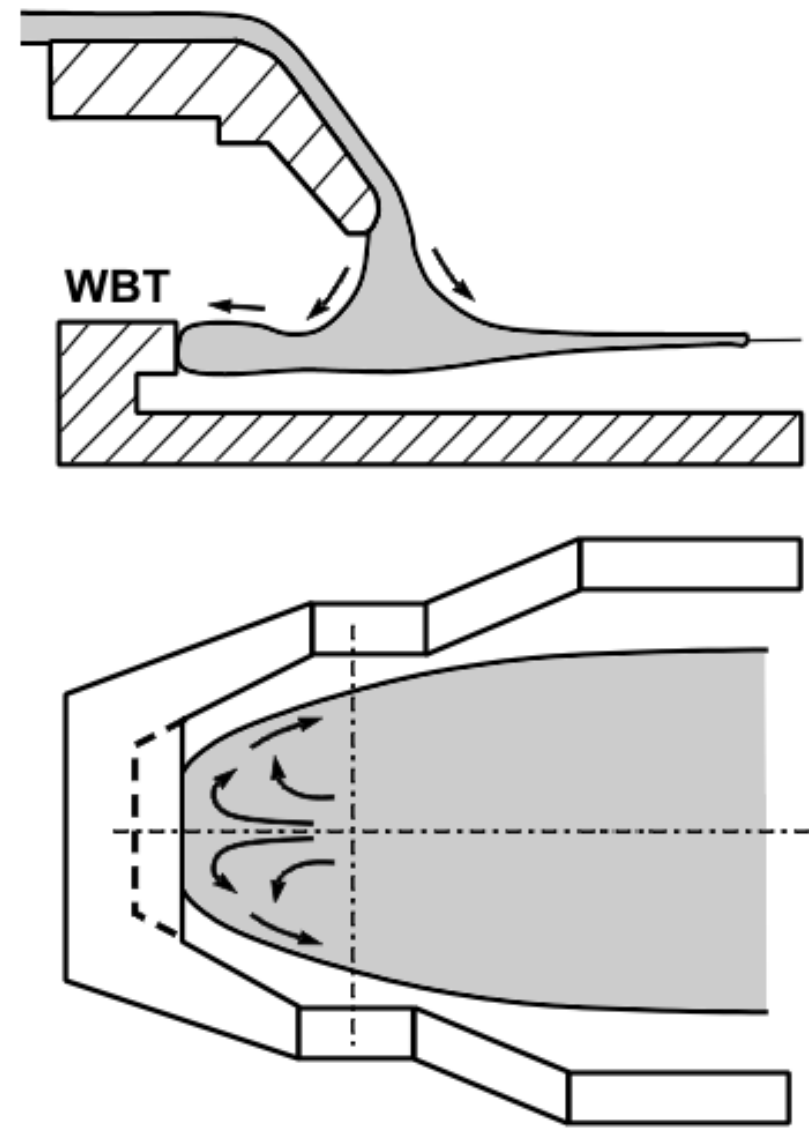

Fig. 6. Wet Back Flow

\section{NUMERICAL MODELLING}

There follows the details of the numerical modelling of the three-phase flow composed of three immiscible, non-reactive fluids separated by one (or more) surfaces known as interphases. Due to the global characteristic of the glass/tin system, the phases are clearly stratified. The glass spreads like in an open-channel flow, supported by the tin bath and driven by gravitational and buoyancy forces. The upper side of the glass ribbon, defining the interface between the gas and the liquid, is a free surface (this designation arises from the great difference between the densities of the gas and the liquid, see table 1). The lower side, or glass/tin interface, is a liquid-liquid interphase. Whatever the type, the presence of these boundary surfaces introduces serious complications. They require the introduction of special methods to define their position, their movement and their influence on the flow.

A method to describe a free surface must present three essential features. First, a scheme is needed to describe the shape and location of a surface. Second, an algorithm is required to track the shape and location in time. And finally, free-surface boundary conditions must be applied at the surface. Different numerical approaches have been developed to deal with interphase modelling. Some techniques are focused on the definition of body-fitted grids that move with the grid (lagrangian schemes). Since the grid is embedded within the fluid, the grid is automatically tracking the interphase. On the other hand, there are eulerian schemes that propose a fixed grid in which the phases are solved over time using transport equations. 
In recent years, different authors have attempted numerical simulations of two-phase flows in both two- and three-dimensional approaches. As a result, a large number of codes (Ripple, Polyflow or Sola-VOF among others) have been designed to obtain accurate results for very specific types of multi-phase problems.

Probably, the most successful of these methods is the volume-of-fluid (VOF) technique because of its simplicity and robustness. It is particularly recommendable for describing highly complex interfaces. In the original algorithm, developed by Hirt and Nichols [27], it is assumed that all the phases (incompressible, viscous fluids) must fill the whole domain, so no void regions are allowed. In addition, to implement the interphase tracking, this algorithm is usually combined with a formulation to include the surface tensions in the momentum equations as volume forces. This algorithm is the continuous surface force (CSF) formulation [28]. An outline of the numerical methods used when combining the VOF and CSF algorithms is provided in the following section.

\section{VOF Model}

The VOF algorithm can model two or more immiscible fluids by solving a single set of momentum equations and tracking the volume fraction of each of the fluids throughout the domain. It is assumed that the flow in each phase is viscous and incompressible. The VOF formulation relies on the fact that two or more fluids (or phases) are not interpenetrating. For each additional phase that is added to the model, a variable is introduced: the volume fraction of the phase in the computational cell, $\alpha_{q}$. In each control volume, the volume fractions of all phases add up to unity. The fields for all variables and properties are shared by the phases and represent volume-averaged values, as long as the volume fraction of each of the phases is known at each location. The tracking of the interface(s) between the phases is accomplished by the solution of a continuity equation for the volume fraction of one (or more) of the phases [29]. For the $q^{\text {th }}$ phase, this equation has the following form:

$$
\frac{\partial \alpha_{q}}{\partial t}+\stackrel{r}{v} \cdot \nabla \alpha_{q}=0
$$

where $q=1,2, \ldots N$ is the total number of phases, $\alpha_{q}$ is the $q^{\text {th }}$ fluid's volume fraction in the cell and $\hat{v}$ is the velocity vector shared by all the phases. Notice that there is no need to resolve the volume fraction equation for all the phases, because there cannot be void regions. In particular, the primary-phase volume fraction will be computed based on the following constraint:

$$
\sum_{q=1}^{N} \alpha_{q}=1
$$

The properties appearing in the transport equations are determined by the presence of the component phases in each control volume. Thus the variables and properties in any given cell are either purely representative of one of the phases, or representative of a mixture of the phases, depending upon the volume fraction values. In general, both volume-fractionaveraged density and viscosity take the following form:

$$
\rho=\sum_{q=1}^{N} \alpha_{q} \rho_{q} \quad \mu=\sum_{q=1}^{N} \alpha_{q} \mu_{q}
$$


A single momentum equation is solved throughout the domain, and the resulting velocity field is shared among the phases. The momentum equation, shown below, is dependent on the volume fractions of all phases through the properties $\rho$ and $\mu$.

$$
\rho \frac{\partial v}{\partial t}+\rho(\stackrel{\mathrm{r}}{v} \cdot \nabla) \stackrel{\mathrm{r}}{v}=\rho \stackrel{\mathrm{r}}{g}-\nabla P+\mu \nabla^{2} \stackrel{\mathrm{r}}{v}+\stackrel{\mathrm{r}}{F_{V}}
$$

The resolution algorithm for equation (4) must be conservative when defining the interphases over time. This means that the numerical diffusion must be strictly controlled when advecting the volume fractions. Basically, all the VOF algorithms compute the variation of the volume fraction $\alpha_{q}$ in a cell by reconstructing the surface from VOF data and computing the volume fluxes geometrically. In particular, two basic methods can be employed: the Simple Line Interface Calculation (SLIC) that approximates the interface with a straight line aligned with the coordinates, or the Piecewise Linear Interface Calculation (PLIC) which allows a linear slope within each cell. The surface reconstruction method plays an important role in the accuracy and robustness on the VOF method. In the case of highly complex interphases, the piecewise-linear approach is the most accurate, being especially recommended for general unstructured meshes.

\section{CSF Formulation}

The VOF model can also include the effects of surface tension along the interface between each pair of phases. Surface tension arises as a result of attracting forces between molecules in a fluid. The surface tension is a force, acting only at the surface, which is required to maintain the equilibrium between the phases. It acts to balance an inter-molecular attracting force with the pressure gradient force across the surface.

In the CSF model, proposed by Brackbill et al. [28], a surface tensile force per unit interphase area (i.e. the surface tension) is replaced by a localized volume force acting on fluid elements located in a this transition region between two phases. This means that surface forces are no longer applied as discrete boundary conditions at a discontinuity (the interphase) but as body forces $\stackrel{1}{F}_{V}$ acting in the transition region.

The real advantage of the CSF formulation is the accurate solution of Laplace-Young equation when reformulated volumetrically. This equation states that the pressure jump across an interphase under surface tension is proportional to the curvature, $\kappa$, of the interphase:

$$
\Delta P=\sigma(-\stackrel{\mathrm{l}}{\nabla} \cdot \stackrel{\mathrm{r}}{n})
$$

where $\sigma$ is the surface tension and the curvature has been defined in terms of the divergence of the unit normal, $\kappa=(-\nabla \cdot r)$. In its standard form, equation (8), surface tension contributes a surface pressure. However, the expression can be rearranged to write a surface force per unit interfacial area [30]:

$$
\stackrel{1}{F}_{S}\left(\stackrel{r}{r_{S}}\right)=\sigma \kappa\left(\stackrel{r}{r_{S}}\right) \stackrel{\mathrm{r}}{n}\left(\stackrel{\mathrm{r}}{r_{S}}\right)
$$


where $\stackrel{\mathrm{r}}{n}\left(\underset{r}{r} r_{S}\right)$ is the unit normal to the surface $(S)$, expressed for a point at the surface ${ }^{r_{S}}$. Note that $\stackrel{1}{F}_{S}\left(\stackrel{\mathrm{r}}{r_{S}}\right)$ corresponds to the pressure acting on the surface $\mathrm{S}:\left|\stackrel{1}{F_{S}}\left(\stackrel{\mathrm{r}}{r_{S}}\right)\right|=\Delta P\left(\stackrel{\mathrm{r}}{r_{S}}\right)$.

The basic idea of the CSF model is that one can define a localized body force, $\stackrel{1}{F}_{V}$, whose volume integral in the limit of infinitesimally small transition-region-thickness $\delta$ is equal to the surface integral of the tensile force, $F_{S}$. Thus [31],

$$
\lim _{\delta \rightarrow \infty} \iiint_{V} \stackrel{1}{F}_{V}(\stackrel{\mathrm{r}}{r}) d V=\iint_{S} \stackrel{\mathbf{1}}{F_{S}}\left(\stackrel{\mathrm{r}}{r_{S}}\right) d S
$$

In order to find the body force $\stackrel{1}{F}_{V}$ that satisfies equation (11), first it is necessary to define a characteristic step function, $c$, that changes discontinuously from one fluid to the other at the interphase. However, to introduce a transition region instead of an infinitesimally thin line, the step function is typically replaced by a smooth variation, defined a B-spline, 80 , over a transition region $\delta$. Mathematically, $8 \not(\stackrel{\mathrm{T}}{r})$ approaches $c(\stackrel{\mathrm{r}}{r})$ as the transition region tends to zero: $\lim _{\delta \rightarrow 0} \&(\underset{r}{\mathrm{r}})=c(\stackrel{\mathrm{r}}{r})$. Using this function $\mathscr{B}(\underset{r}{\mathrm{r}})$, normalized with $[c]=c_{2}-c_{1}$, it is possible to find a distribution for the volume force $F_{V}$ that satisfies the integral equation (10) in the following form [32]:

$$
\stackrel{\mathrm{r}}{F_{V}}(\stackrel{\mathrm{r}}{r})=\sigma \kappa(\stackrel{\mathrm{r}}{r}) \frac{\nabla \&(\stackrel{\mathrm{r}}{r})}{[c]}
$$

In addition, since the isovalues of the smooth function represent the progressive change from one phase to the other (i.e., the interphase), the unit normal to that surface can be

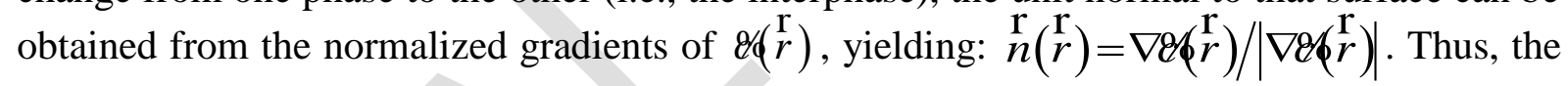
curvature becomes:

$$
\kappa(\stackrel{\mathrm{r}}{r})=-\nabla \cdot\left(\frac{\nabla \otimes_{(}(\stackrel{\mathrm{r}}{r})}{\left|\nabla \&\left({ }^{\mathrm{r}} r\right)\right|}\right)
$$

Equation (12) introduced in equation (11) provides a compact definition of the body forces, only depending on the smooth function. Note that $F_{V}$ is not a function of the surface location, $r_{S}$; rather, it is a true volume force that depends on $\stackrel{1}{r}$. The discrete boundary condition is replaced by a continuous model. Since $\mathcal{B}(r)$ is constant in each fluid and changes only within the transition region, $\stackrel{1}{F}_{V}$ becomes zero outside the interphase region. Obviously, $\stackrel{1}{F}_{V}$ can be directly added to the momentum equation (7).

Finally, the CSF formulation is completely combined with the VOF model replacing the smooth step function with the volume fraction of the phases:

$$
\mathcal{E}(\stackrel{\mathrm{r}}{r})=\alpha_{q}(\stackrel{\mathrm{r}}{r})=\left\{\begin{array}{cc}
0 & \text { outside phase- } \mathrm{q} \\
0<\alpha_{q}<1 & \text { in the interphase } \\
1 & \text { inside phase- } \mathrm{q}
\end{array}\right.
$$


Therefore, for two-phase flows, the source term to introduce in the momentum equation results from the equations (11) through (13) as:

$$
\stackrel{\mathrm{r}}{F_{V}}=-\sigma_{i j} \rho\left[\nabla \cdot\left(\frac{\nabla \alpha_{i}}{\left|\nabla \alpha_{i}\right|}\right)\right] \frac{\nabla \alpha_{i}}{\left(\rho_{i}+\rho_{j}\right) / 2}
$$

where $\rho$ is the volume-fraction-averaged density according to (6) and $[c]$ has been replaced by the averaged density of both fluids. In the case of more that two fluids, this equation can be generalized as a superimposition of forces for every pair of phases immersed in a cell:



The model can be augmented by the additional specification of the contact angles between the phases and the walls. If the fluid interphase forms a contact angle $\phi$ with a rigid boundary that differs from the equilibrium one $\left(\phi_{w}\right)$, a resulting force should be applied in order to restore the fluid to the equilibrium contact. This force can easily be computed within the framework of the CSF technique by applying a wall adhesion BC, parallel to the wall, according to $F_{w}=\sigma\left(\cos \phi-\cos \phi_{w}\right)$.

\section{Numerical stability}

The VOF model can resolve stratified flows of immiscible fluids, either implicitly or explicitly depending on the nature of the flow. If the process varies over time as well as in space, a time-accurate transient behaviour of the VOF solution is required. Hence, when it is necessary to track the evolution of the interphases, a time-dependent, explicit interpolation scheme must be used. Unfortunately, the process of solving a multiphase system is inherently difficult, especially in the case of a time-marching solution strategy. Most of the time, stability and convergence problems arise during the resolution, so severe limitations must be fixed in the numerical scheme.

Mainly, the basic restriction is related to the time step used for the volume fraction calculation, which is severely restricted by different parameters. In particular, the physical properties of the phases (density and viscosity), the transient velocity of the interphase(s) and the mesh density are the critical factors. Also, there are three important stability criteria, related to the governing equations, that must be satisfied at a time. Each of them will provide a time step constraint regarding the momentum and VOF advection, the viscous terms and the body forces (reformulation of the surface tension) [33].

The first criterion is related with the convective fluxes for a fluid element across a control volume. Actually, this condition specifies that a fluid cannot move more than one cell in one time step. Then, the time step should be lower that the time needed for the fluid to empty out of the cell (in any direction), yielding the classic Courant condition (CFL condition): 


$$
\Delta t<\min \left(C \frac{\Delta x}{|u|}, C \frac{\Delta y}{|v|}, C \frac{\Delta z}{|w|}\right)
$$

where $C$ is the Courant number, taking values between 0 and 1 . Note that lower Courant numbers provide more stability (more conservative condition), but reduce notably the time step. This is a pure kinematic restriction, linked to the interphase velocity and the cell size of the grid. If this condition is violated, the interphase tracking is lost and the solution diverges. A second stability criterion refers to the momentum transfer involved in the viscous terms of the equations. In this case, momentum must not diffuse more than one cell in one time step for every phase. This dynamic condition, similar to the previous kinematic relation, is evaluated as:

$$
\Delta t<\frac{1}{3} \frac{\rho_{q}}{\mu_{q}}\left(\frac{(\Delta x \Delta y \Delta z)^{2}}{\Delta x^{2}+\Delta y^{2}+\Delta z^{2}}\right)_{\min }^{2 / 3} \forall q
$$

The physical properties of the phases, combined with the grid density, constitute the real constraint for the time step.

The third criterion should be considered only if surface tension effects are relevant. The explicit treatment of surface tension is stable when the time step resolves the propagation of capillary waves on the surface. In the case of a uniform, rectangular grid, using a conservative maximum phase velocity that corresponds to minimum resolvable wavelength [28], this yields:

$$
\Delta t<\left[\frac{\left(\rho_{i}+\rho_{j}\right) \cdot \min (\Delta x \Delta y \Delta z)}{4 \pi \sigma_{i j}}\right]^{1 / 2}
$$

This final constraint prevents capillary waves from travelling more than one cell width in one time step. It depends on the value of the surface tension for both phases $i, j$. Obviously, in the case that surface tension is negligible, this third restriction vanishes.

To assure the stability, the time step for the time-marching solution must be lower than the lowest time step calculated from equations (16) to (18). Also, these restrictions show that the mesh density is a very important parameter that influences the election of an accurate time step. The denser the grid, the lower the time step must be to fulfil the restrictions. Moreover, if the number of cells is large, not only does the time step have to be reduced, but also the number of iterations needed to converge as well as the CPU time per iteration is dramatically increased. Therefore, it is extremely important to find an optimum equilibrium between the needed spatial discretization (function of the desired resolution) and the CPU time required to complete the simulation (depending on the computational resources, the deadline times, the number of cases, etc...)

\section{A TEST CASE: THE TWO-DIMENSIONAL APPROACH.}

The commercial package FLUENT 6.0 [34] has been used to solve the set of NavierStokes equations and the VOF algorithm with CSF reformulation. The equations are discretized using the finite volume method on a co-located grid in a first-order fully implicit, unsteady form. Since the flow is dominated by body forces rather than pressure gradients, a 
body-forced weighted discretization is used for the diffusion terms in the pressure interpolation scheme. Turbulence modelling has been considered through an anisotropic RSM scheme, due to the turbulent regime of the tin phase. The second order upwind differencing scheme has been used for convective terms in the momentum equations and also in the terms for the turbulent variables $k, \varepsilon$ and the Reynolds stresses. In addition, the volume fraction equations have been discretized with a geometric reconstruct scheme, using a piecewise-linear approach (PLIC) to represent the interphase between the fluids. The PISO algorithm was used for coupling the pressure and velocity terms, recommended in the case of transient calculations for multiphase flows. Most of the under-relaxation factors were reduced to values between 0.2 and 0.5 to improve the convergence history.

Implicit first-order numerical scheme is employed for time stepping. Simulations were initiated with very small time steps $\left(\sim 10^{-4}\right)$ to be increased gradually for later times with no spurious numerical oscillations in the solution. The volume-of-fluid sub-time step is calculated internally based on the mesh resolution and fluxes in the interface cells -equations (16) through (18)-. VOF sub-time step determines the minimum time to move the outer fluid out of an interface cell. It is recommended using a time step no more than 10-20 times larger than the VOF sub-time step for stable simulations.

\section{Two-dimensional geometry}

The present section develops a two-dimensional approach of the pouring glass process and its further spreading out over the tin surface of an industrial float. Figure 7 shows a threedimensional view of the start area of the float. This trapezoidal, funnel-shaped area is 1.5 meters long (from the spout lip to the restrictor tiles end zone), 0.5 meters high, and progressively wider, going from 1 meter wide at the spout lip to almost 4 meters at the end of the restrictor tiles. The two-dimensional test case, implemented to determine the optimum numerical parameters of the simulation, considers the longitudinal plane of symmetry of the float (grey plane in the figure).

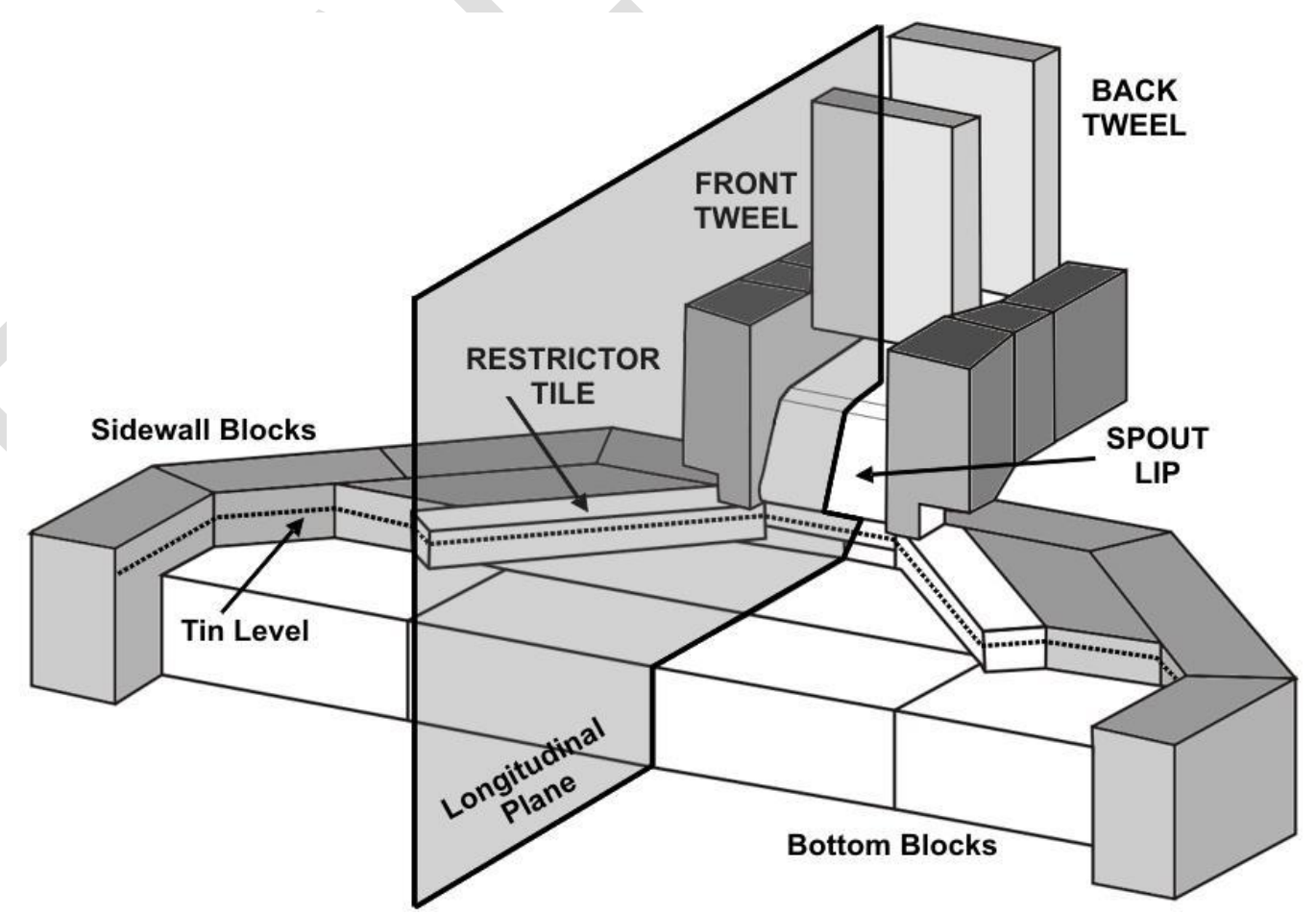

Fig. 7. Longitudinal plane of symmetry. 
A representation of the computational domain is shown in figure 8. A structured grid, with adaptative gridding patterns to improve the description of the tin's free surface, has been introduced in the model. Different meshes were tested in order to obtain a reasonable ratio between the spatial description of the flow and the computational cost of the simulation. Note that if the mesh is extremely dense, the time restrictions impose an extremely low time step, delaying the resolution. On the contrary, coarse meshes may lead to poor spatial definition of the interphases or even inaccurate solutions [35]. For this test case, a moderate mesh with approximately 7,850 cells was adopted. Excluding the mesh around the spout lip, the cell distribution in both horizontal and vertical directions is [140x50] respectively. Notice that the grid density is concentrated beneath the spout lip, since it is expected that higher gradients of the variables will be established there. In that zone, minimum cell sizes are $\Delta x=3 \mathrm{~mm}$ and $\Delta y=1.5 \mathrm{~mm}$. From thereafter, the grid points are progressively separated in order to economize the total cell number.

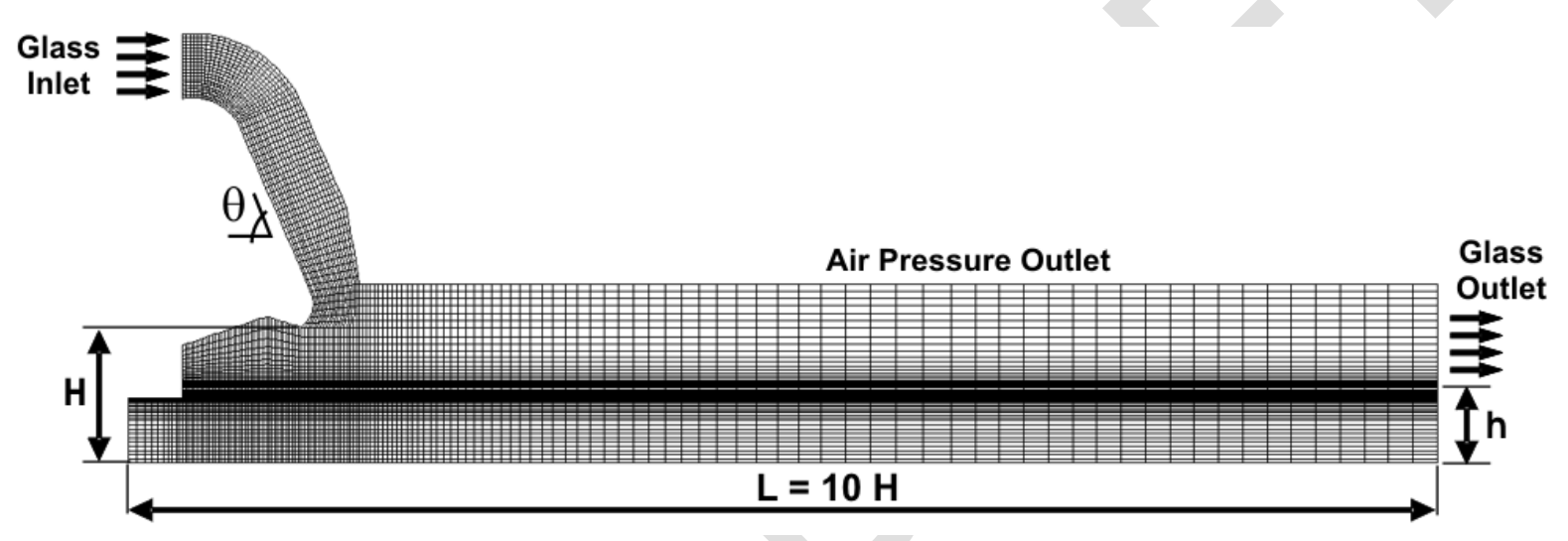

Fig. 8. Two-dimensional mesh.

Boundary conditions are also specified in figure 8. A horizontal pressure-outlet condition is defined for the outgoing air that must leave the domain as the incoming glass is replacing it. This boundary condition is horizontal in order to specify a constant atmospheric pressure for the air phase. At the inlet, a uniform velocity is imposed for the glass phase. The size of this condition is in the range of the expected thickness of the glass film moving down the spout lip -according to equation (1). Finally, an outlet condition for the mass flow rate (glass outlet in the figure) is placed well downstream (at ten times the height of the vertex of the spout above the ground level, H) to avoid any influence on the glass ribbon formation. The remaining boundaries are prescribed as solid walls. In addition, the resolution is started including the tin level in the initialization (at height $h$ ), patching the cell zones that are flooded with molten tin. The tin phase will remain at rest until the glass begins to spread out onto the free surface of the bath. After the contact between the phases is established, the molten tin will be sheared by the glass ribbon.

The solution is initiated and allowed to proceed until both glass and tin phases are steady-stated. This is observed from the variation of the outlet mass flow rate of glass as a function of time. Once the flow becomes steady, the position of the interphases and the velocity fields are definitively known. With the mesh density of figure 8, typical CPU times of 125 hours were needed to simulate around 75 seconds until obtaining the steady-state solution in a Pentium III @ 800 Mhz. This high computational cost is a direct consequence of 
the large number of discretized equations, along with the reduced time steps that are usually necessary to fulfil convergence.

\section{Numerical parameters}

The isothermal and incompressible Navier-Stokes set of equations, combined with a three-phase VOF formulation including CSF reformulation, are resolved numerically. This methodology has been tested as an effective tool for studying engineering problems with multiple free surfaces under non-hydrostatic pressure distributions [36]. No mechanical input of the top rollers is considered in the model. Hence, these assumptions allow simulating the glass pouring process without thermal gradients as the ribbon reaches the equilibrium thickness. This means that the simulation is valid only in the entrance area of the float, from the wet back tile to the end zone of the restrictor tiles. From there on, the glass, progressively cooled, changes its rheological properties, and the top rolls modify the ribbon thickness.

Table 1 shows the physical parameters of the all the phases involved in the process. The values for the glass are taken at $1100^{\circ} \mathrm{C}$, the temperature in the float entrance area. Its Reynolds number, based on the equilibrium thickness for a mass flow rate of 550 ton/day, is clearly below unity confirming its laminar behaviour. On the contrary, the tin's Reynolds number, based on the bath depth and the ribbon velocity, is within the turbulent range. Typical values for the turbulent parameters have been established for both air and tin phases in the boundary conditions according to standard relationships between $k$ and $\varepsilon$. In addition, the importance of surface tension effects is determined based on the value of two dimensionless numbers: the capillary $(\mathrm{Ca})$ number when $\operatorname{Re}=1$ and the Weber $(W e)$ number when $\operatorname{Re}$ ? 1. As a general rule, it is assumed that the surface tension effects can be neglected if the capillary or the Weber numbers are greater than one:

$$
C a=\frac{\mu U}{\sigma} ? 1 \quad W e=\frac{\rho L U^{2}}{\sigma} ? 1
$$

In the case of the glass, the capillary number is 12 , while for the tin phase, the Weber number is 20. Both non-dimensional numbers are approximately of the order of the unit, so surface tension will show a significant influence on the flow dynamics of the phases.

\begin{tabular}{|c|c|c|c|}
\hline & GLASS & TIN & AIR \\
\hline \multicolumn{4}{|l|}{ Physical Parameters } \\
\hline $\begin{array}{l}\text { Density }\left[\mathrm{kg} / \mathrm{m}^{3}\right] \\
\text { Viscosity }[\mathrm{Pa} . \mathrm{s}] \\
\text { Surface Tension }[\mathrm{N} / \mathrm{m}] \\
\end{array}$ & $\begin{array}{c}2500 \\
100 \\
0.5 \\
\end{array}$ & $\begin{array}{l}6770 \\
0.001 \\
0.35 \\
\end{array}$ & $\begin{array}{c}1.225 \\
1.8 \cdot 10^{-05} \\
0.5(G-T) \\
\end{array}$ \\
\hline \multicolumn{4}{|l|}{ Turbulence Parameters } \\
\hline $\begin{array}{l}\text { Turb. Kinetic Energy }\left[\mathrm{m}^{2} / \mathrm{s}^{2}\right] \\
\text { Turb. Dissip. Rate }\left[\mathrm{m}^{2} / \mathrm{s}^{2}\right]\end{array}$ & - & $\begin{array}{c}2.4 \cdot 10^{-05} \\
4 \cdot 10^{-05} \\
\end{array}$ & $\begin{array}{c}1 \\
4 \cdot 10^{-05} \\
\end{array}$ \\
\hline \multicolumn{4}{|l|}{ Non-dimensional Parameters } \\
\hline $\begin{array}{l}\text { Reynolds number }[-] \\
\text { Weber number }[-] \\
\text { Capillary number }[-]\end{array}$ & $\begin{array}{l}0.0175 \\
0.35 \\
20\end{array}$ & $\begin{array}{l}60000 \\
12 \\
2 \cdot 10^{-04}\end{array}$ & $\begin{array}{l}- \\
- \\
-\end{array}$ \\
\hline
\end{tabular}

Table 1. Physical parameters. 
These calculations have been performed with a time step of 0.02 seconds, which satisfies the restrictions summarized in equations (16) to (18) for a Courant number of 0.75 in the sub-time stepping of the VOF model. Also, 75 iterations per time step were fixed in the early stages of the simulation. When the flow is close to being fully-developed, the number of iterations per time step can be notably decreased with no loss of accuracy in the convergence history. It is even possible to increase the size of the time step if the interphases are practically steady-stated.

In the following section, results of the two-dimensional approach of the float for different operating conditions are presented. In particular, a typical mass flow rate of 550 ton/day is analyzed, denoted as $Q_{0}$, as well as higher and lower conditions, ranging from $150 \%$ and $125 \%$ to $75 \%$ and $50 \%$ of the reference flow rate $Q_{0}$.

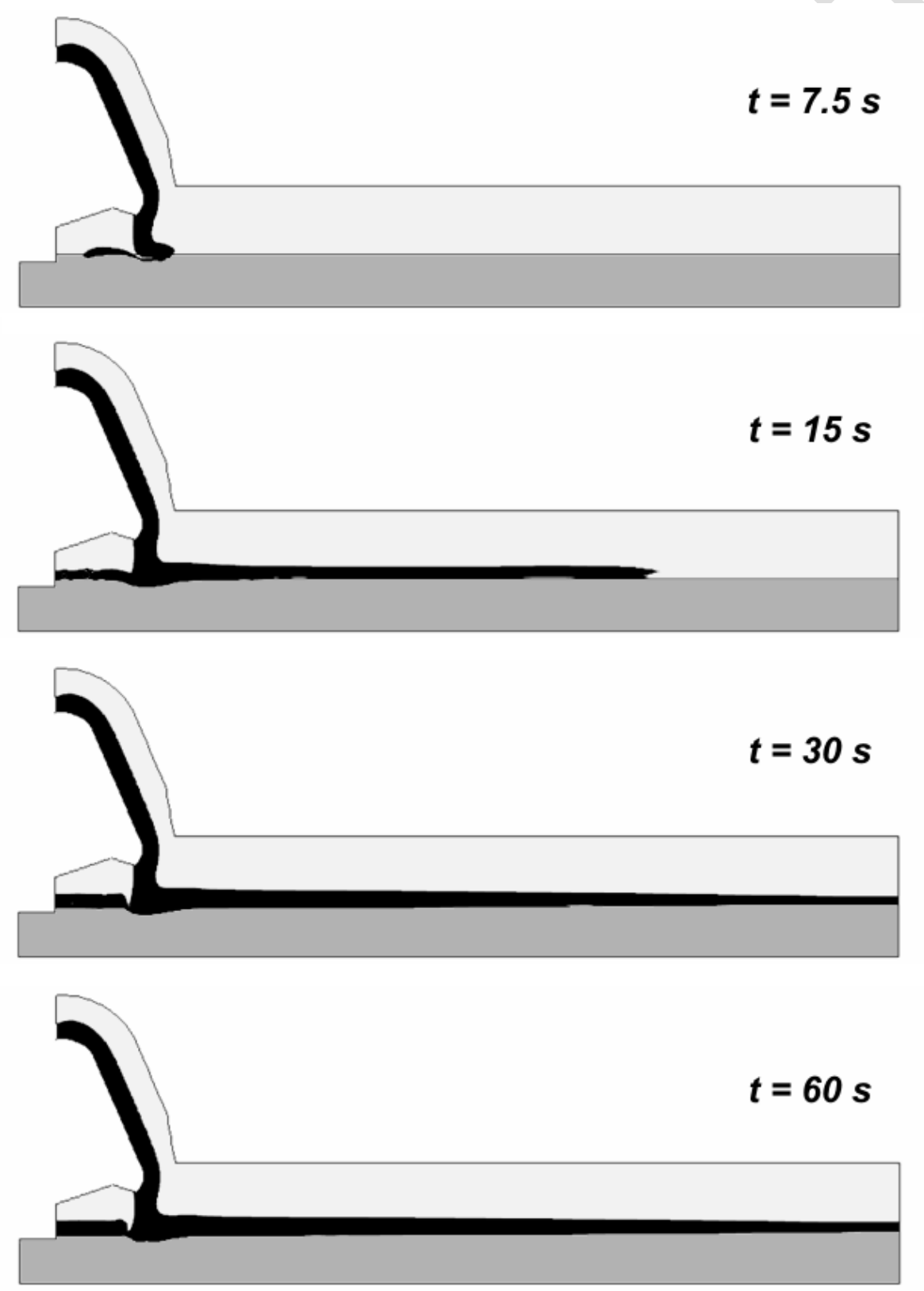

Fig. 9. Temporal evolution of the simulation. 


\section{Results and discussion}

Figure 9 shows the time-marching philosophy of the unsteady numerical model in the case of the nominal flow rate $\left(Q_{0}\right)$. At the start of the simulation, $t=0$, the glass begins to advance progressively towards the end of the spout lip, replacing the air phase (in light grey) on the way. When 7.5 seconds have passed, the glass (black region) has already been poured onto the tin's surface, with a small part flowing backward into the wetback region forming an incipient "wet back flow". After 15 seconds, the glass ribbon is clearly established, spreading out towards the downstream outlet. Also, the pool of glass trapped beneath the spout lip in the wetback region thickens. The ribbon, floating on the molten tin (in dark grey), is progressively flattened due to the mechanisms of equilibrium thickness, as observed at $t=30 \mathrm{~s}$. Note that the interphases are practically stable at that point, so the simulation is very close to a steady-state condition. In fact, it is at $t=60 \mathrm{~s}$ when it can be considered that the transient stage of the simulation is over. The glass depth beneath the pouring spot or thickness of the wet back flow does not suffer any variation over time. Hence, both inlet and outlet glass flow rates are perfectly balanced and the definitive position of the interphases is known.

In order to define an accurate methodology, it is necessary to provide a quantitative criterion to determine when the steady-state is established, instead of doing it qualitatively as in figure 9. Basically, the proposed procedure consists of declaring the fully-developed state of the pouring process when the outgoing flux of glass equals the flow inlet.



Fig. 10. Steady-state solutions.

This can be observed in the history of the variation of the glass flow rate at the outlet, shown in figure 10. This figure reveals the results for the database considered in this test case: $1.5 \times Q_{o}, 1.25 \times Q_{o}, Q_{o}, 0.75 \mathrm{xQ}_{0}$ and $0.5 \mathrm{xQ}_{\mathrm{o}}$. The $x$-axis represents the time elapsed from the simulation start-up, while the $y$-axis contains the equilibrium value for every case. Due to numerical diffusion and truncation errors, the outlet flow of glass cannot exactly match the inlet conditions. Then, the maximum accuracy is obtained when the outlet flow varies steadily 
with a determined interval of confidence, typically $95 \%$. All the cases required at least 25 seconds for the glass to reach the domain exit. From then on, the simulation was extended until the variations of the outgoing glass followed a controlled, in-range evolution. After 50 seconds, all the cases present a confirmed steady-state. The exact length of the transient period cannot be determined a priori, so it must be analyzed separately for every case. Anyway, the higher the mass flow rate, the more glass is accumulated inside the domain, so higher stabilization times are required.

Now, we present encouraging results obtained from the simulation for the nominal case. For instance, figure 11 illustrates in detail the two-dimensional distribution of the velocity fields for both glass and tin phases. Over the spout lip, the glass behaves as a thin film of oil flowing down towards the tin in a laminar regime. The velocity profile, superimposed on the glass ribbon (black contour of the volume fraction of glass), presents a parabolic shape as expected from equation (2). On the contrary, the glass sheet over the tin bath, exhibits a clear uniform profile. This effect is a direct consequence of the extremely low shear stress that both air and tin phases impose at the glass interphase, because of the reduced viscosity of both fluids (table 1). On the other hand, the glass/tin momentum transfer established at the interphase, pulls the upper layers of tin (see the velocity vectors in figure 11). This effect is compensated with an inner recirculation of molten tin, generated in the lower zones of the bath. Beneath the lip, the velocity distributions change rapidly because the flow cannot be fully developed there. Also notice how the typical velocity values in the wet back region are extremely low, increasing the residence times for both glass and tin in that zones.



Fig. 11. Velocity profiles in the glass ribbon and the tin bath.

Further details of the glass ribbon moving onto the tin's surface are included in figure 12. The figure shows the velocity distribution at the end zone of the restrictor tiles 
(approximately 1.5 meters downstream the spout lip). Once the flow is fully developed, the glass thickness is practically constant all along the length of the bath. Also, no significant variations of the velocity field in the tin phase are observed. Since the tin is confined in the bath, recirculation currents are a result of the shear flow occurring beneath the glass ribbon.



Fig. 12. Mometum transfer between the glass ribbon and the tin bath.

Finally, figure 13 compares the ribbon characteristics at three different locations for the set of simulated operating conditions. In particular, the glass thickness and the ribbon velocity are analyzed in a section over the spout lip (section (1) in figure 13) as well as in the end section of the restrictor tiles (section (2) in the figure). The velocity magnitudes are made non-dimensional by the velocity value of the reference case, while the thickness is adimensionalized by the tin level. Also, the maximum glass depth beneath the spout lip is presented in the table-zone (3). The results exhibit a non-linear, up-going trend of these variables as the mass flow rate is increased, with maximum values for the glass depth around a $20 \%$ of the initial tin level. These numerical trends are consistent with the observations of the manufacturer in their typical industrial floats. However, though expected, these results are overestimated due to the lack of three-dimensional effects in this $2 \mathrm{D}$ approach. The next section shows how the sidewall boundary conditions of the float and the lateral passages with upstream, recirculated tin currents dramatically modify the fluid dynamics of the tin, reducing the velocity profiles in the longitudinal symmetry plane. 


\begin{tabular}{|c|c|c|c|c|c|}
\hline & \multicolumn{5}{|c|}{ GLASS FLOW RATE } \\
\hline & $0.5 Q_{0}$ & $0.75 Q_{0}$ & $Q_{0}$ & $1.25 Q_{0}$ & $1.5 Q_{0}$ \\
\hline \multicolumn{6}{|l|}{ Lip Section (1) } \\
\hline Dimensionless Glass Thickness & 0.28 & 0.33 & 0.36 & 0.39 & 0.41 \\
\hline Ribbon Velocity $(\mathrm{cm} / \mathrm{s})$ & 0.58 & 0.81 & 1.00 & 1.17 & 1.34 \\
\hline \multicolumn{6}{|l|}{ Exit of RT Section (2) } \\
\hline Dimensionless Glass Thickness & 0.22 & 0.26 & 0.30 & 0.34 & 0.38 \\
\hline Ribbon Velocity $(\mathrm{cm} / \mathrm{s})$ & 0.60 & 0.80 & 1.00 & 1.10 & 1.19 \\
\hline $\begin{array}{l}\text { Dimensionless } \\
\text { Maximum Glass Depth (3) }\end{array}$ & 0.16 & 0.18 & 0.21 & 0.23 & 0.26 \\
\hline
\end{tabular}

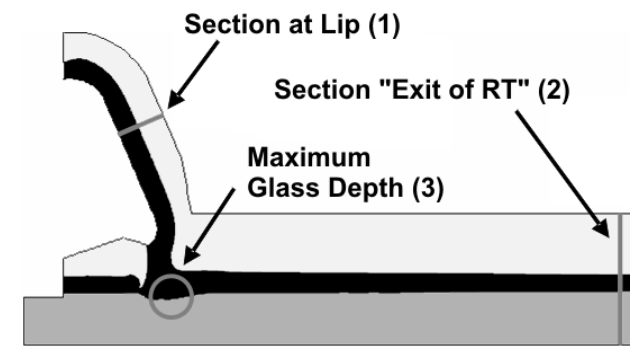

Fig. 13. Results for different operating conditions.

\section{THREE-DIMENSIONAL APPROACH}

The $2 \mathrm{D}$ test case has been introduced as the starting point for a complete threedimensional simulation of the glass pouring process. Basic numerical parameters have been fixed through the two-dimensional approach. Also, overall trends describing the performance of a float have been presented. Local flow phenomena were even reproduced with reasonable discrepancies. However, neither the equilibrium thickness nor the wet back flow has been perfectly adjusted in the modelling.

The lack of a complete description of the process led us to attempt a full 3D unsteady simulation of the three-phase flow. A three-dimensional model allows the analysis of transversal gradients in the phases and introduces the effects of lateral sidewalls (casing) in the flow. The numerical scheme of the 3D model reproduced exactly all the characteristics of the 2D simulation. Also, the transient strategy to resolve the steady-state of the process has been followed. Other assumptions, like isothermal flow with constant physical properties of the fluids are preserved.

\section{Three-dimensional geometry}

The present geometry reproduces the basic dimensions of the entrance area of the float bath in the Saint-Gobain glass manufacturing plant in Avilés (northern Spain). For simplicity's sake, only essential geometric details have been reproduced in the modelling. The three-dimensional domain has been divided into regular, paralepipedic volumes in order to facilitate meshing operations with structure, progressive grids that prevent cell skewness. Also, a reduced, symmetric domain has been considered due to both symmetric operating and constructive conditions of the float (figure 3). Obviously, this implies a notable reduction in the total number of cells, allowing the refinement of specific zones of interest (i.e., the free surface of the bath).

The spatial discretization of the $2 \mathrm{D}$ case was adopted for the symmetry plane of the domain. Afterwards, this mesh distribution was transversally extruded to cover the $z$-axis. A coarser grid was defined in this direction to prevent an excessive number of cells, resulting in a cell for every $20 \mathrm{~mm}$. Total mesh size is [140x50x27], approximately 190,000 cells. Figure 14 shows the progressive characteristics of the grid, including the definition of the boundary conditions. 


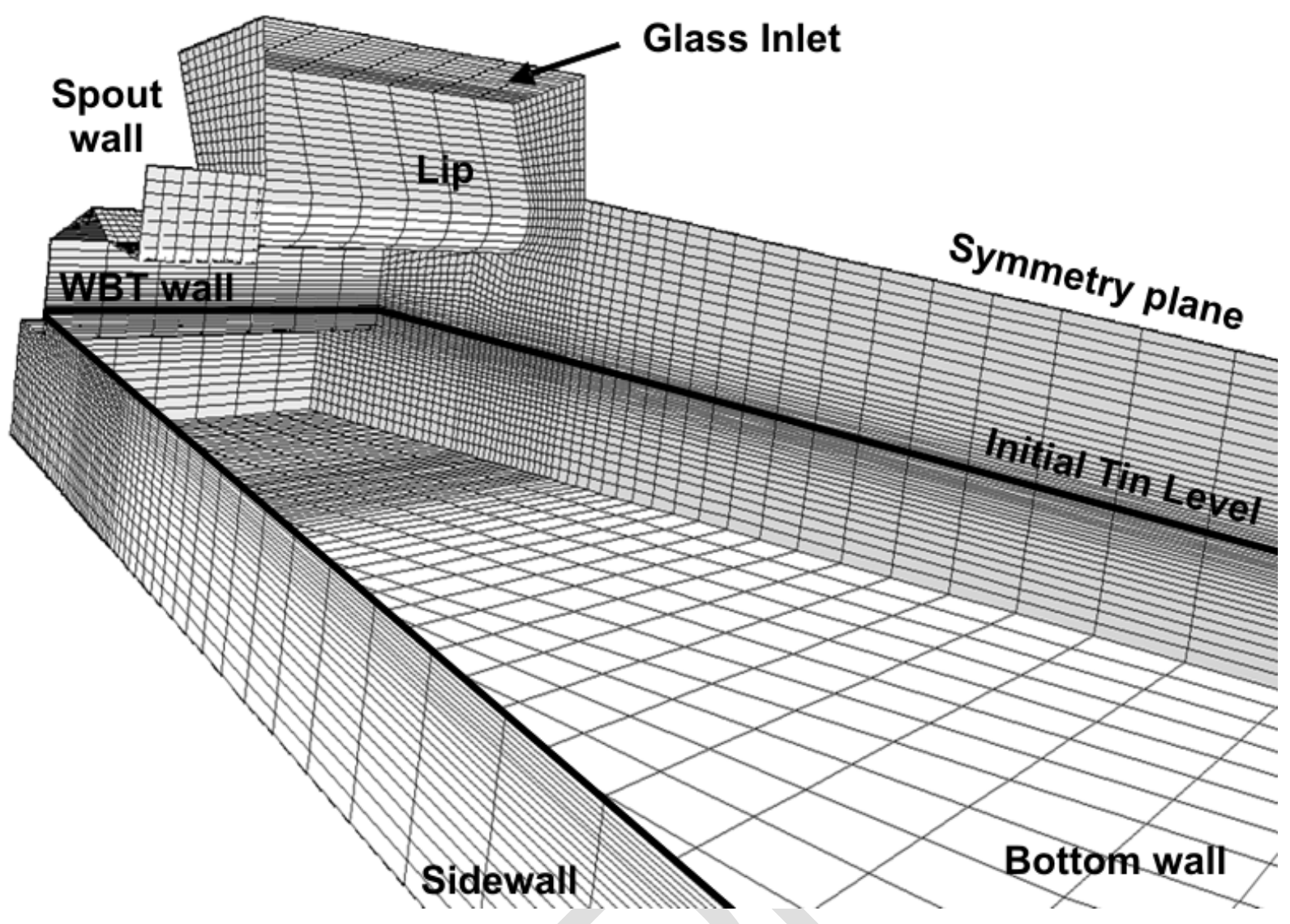

Fig. 14. Three-dimensional mesh.

\section{Numerical parameters}

The restrictions for the time step are slightly modified in the case of this threedimensional grid. Thus, a time step of $0.01 \mathrm{~s}$ has been recalculated to assure numerical stability. The CPU time is severely increased for three-dimensional simulations. In this case, not only the total number of equations to resolve is increased, but also the transient simulation is enlarged. To fulfil these requirements, the simulation was executed using parallel computing over a 10 PC cluster @ 1.2 GHz. More than 500 hours of CPU time were needed to compute a 30 seconds simulation. The rest of the basic parameters, like characteristic velocities and length scales or the physical properties of the phases, are maintained from the $2 \mathrm{D}$ case.

Figure 15 illustrates how the glass spreads out onto the molten tin. A duplicated view of the domain is obtained using the symmetry condition as a mirror plane. The advance of the meniscus of the glass ribbon is perfectly described, with a typical curved wavefront: on the sidewalls, the ribbon is attained by the shear stresses of the lateral casings, so the glass moves faster in the centre of the bath. Also notice the three-dimensional closure of the glass ribbon beneath the spout wall. There, despite of the pouring process, the glass phase does not break up in droplets, thus ensuring a continuous ribbon as required. 


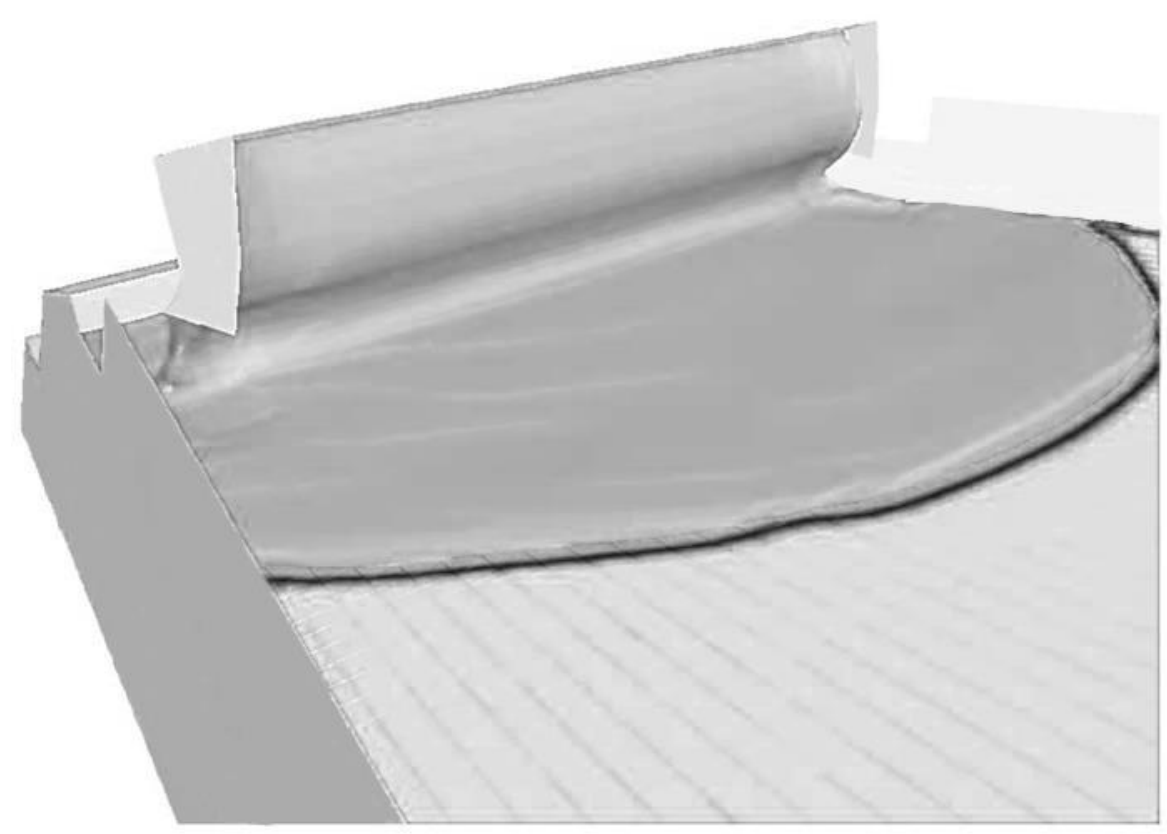

Fig. 15. 3D view of the glass ribbon.

\section{Results}

The same database of the 2D approach has been reproduced for the full 3D model. Figure 16 compares the geometric profile of the glass ribbon in the central plane of the float for all the mass flow rates tested in the simulations. Both axes represent the streamwise and vertical directions respectively. They have been made non-dimensional with the distance between the bottom wall of the bath and the end vertex of the nose of the spout lip, denoted as $H$. These results confirm previous analysis derived from the two-dimensional approach: when the mass flow rate is increased, the glass ribbon thickens, especially in the upper interphase (glass/air interphase). Also, the pool of glass trapped beneath the spout lip is thickened, indicating a progressive accumulation of glass. When the float operates at reduced flow rates (i.e. $0.5 \mathrm{x} \mathrm{Q}_{\mathrm{o}}$ ), the glass film is especially influenced by the shape of the spout lip, showing a clear tendency to stay attached to the convex surface of the nose. This is a clear contribution of the surface tension effects that dominate when the glass thickness is drastically reduced. Moreover, due to the inward curvature of the nose, the boundary layer attachment forces the glass to move upstream. This mechanism may lead to a severe reinforcement of the wet back flow, compromising the quality of the glass. Furthermore, progressive reductions on the glass flow rate may even lead to break up of the continuous ribbon, due to an excessive reduction of the film thickness. On the contrary, large flow rates produce the establishment of thicker ribbons that fall almost vertically from the lip. If the flow rate is excessively increased, the influence of the nose completely vanishes with a ribbon spreading out like a jet that impinges normally on the tin's surface. Therefore, a large amount of glass would be forced to move upwards, also increasing the wet back flow. In any case, the simulations confirm that operating conditions outside the nominal range may produce an increase of the wet back flow. This suggests that it is possible to find an optimal geometry of the spout lip as a function of the glass rate and its physical properties (density and viscosity) that minimizes the impact of the wet back flow. Hence, the distance between the nose and surface of the tin, the shape and curvature of its end vertex and the inclination angle of the lip could be determined numerically to provide an optimal performance for different flow rate conditions. 


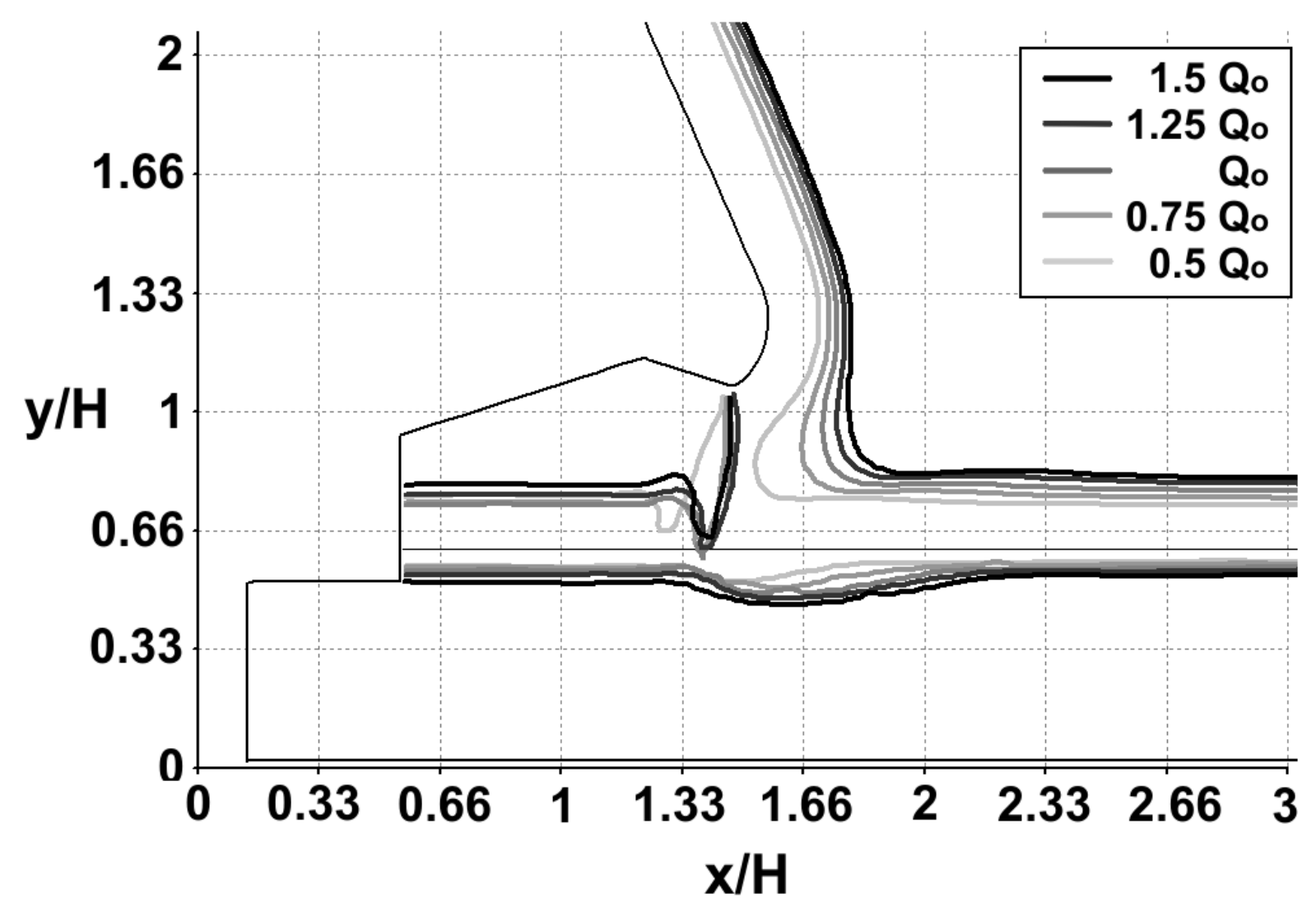

Fig. 16. Geometric profiles for different operating conditions.

Other important results of the simulation refer to the glass profiles at the end zones of the restrictor tiles. Figure 17 compares the transversal variation of the ribbon thickness as a function of the mass flow rate. The transversal distance has been made non-dimensional with the distance $\mathrm{H}$ as usual, while the ribbon thickness is adimensionalized with respect to the tin level, h. The figure is completed with the transversal distributions of the streamwise velocity of the glass ribbon. It is a common feature for all the cases that the glass ribbon is slightly thicker at central positions. This is an additional effect of the friction with the sidewalls. Besides, all cases exhibit a clear asymptotic tendency towards the equilibrium thickness. On the other hand, the velocity profiles present a parabolic behavior that tends to zero due to the zero-velocity condition in the casing.

Figure 18 shows vertical distributions of the streamwise velocity for both glass and tin phases at the restrictor tile exit. The dimensionless results of the complete database are compared. The extremely high viscosity of the glass is responsible for the uniform velocity distribution of the ribbon in all the cases. Typical velocities for the glass ribbon, around 10 $\mathrm{cm} / \mathrm{s}$, are obtained in the simulation, in reasonable agreement with the real velocities measured in the float [4]. Also notice that the higher the mass flow rate, the larger the streamwise velocity, confirming the convergent tendency towards the equilibrium thickness for all the cases. In particular, this is observed in the tangent limits of the uniform distributions of the ribbons. On the other hand, the velocity distribution of the tin currents shows that the recirculation beneath the glass ribbon is significantly lower than those predicted by the two-dimensional approach. This is a direct consequence of the transversal redistribution of the recirculated tin currents in the 3D model. Moreover, in the central plane, the tin velocity roughly presents negative values (i.e. upstream currents), so the recirculation circuit for the confined tin is established through the lateral passages. This observation is a 
classic characteristic, a well-known flow pattern of any industrial float [23], also captured by this numerical modelling.

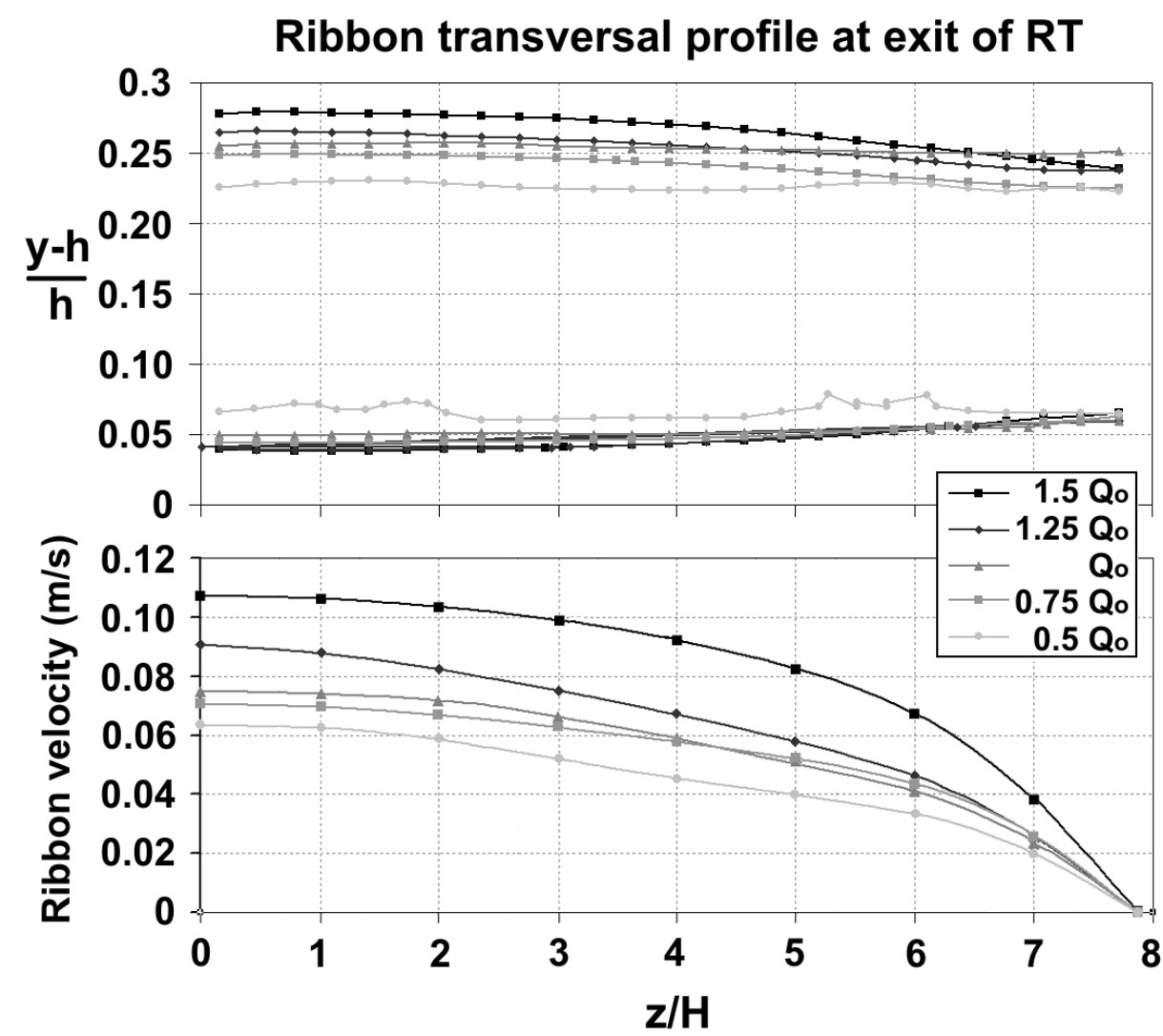

Fig. 17. Transversal profiles of the glass ribbon for different flow rates.

\section{Velocity Distributions in the bath centerline at exit of RT}
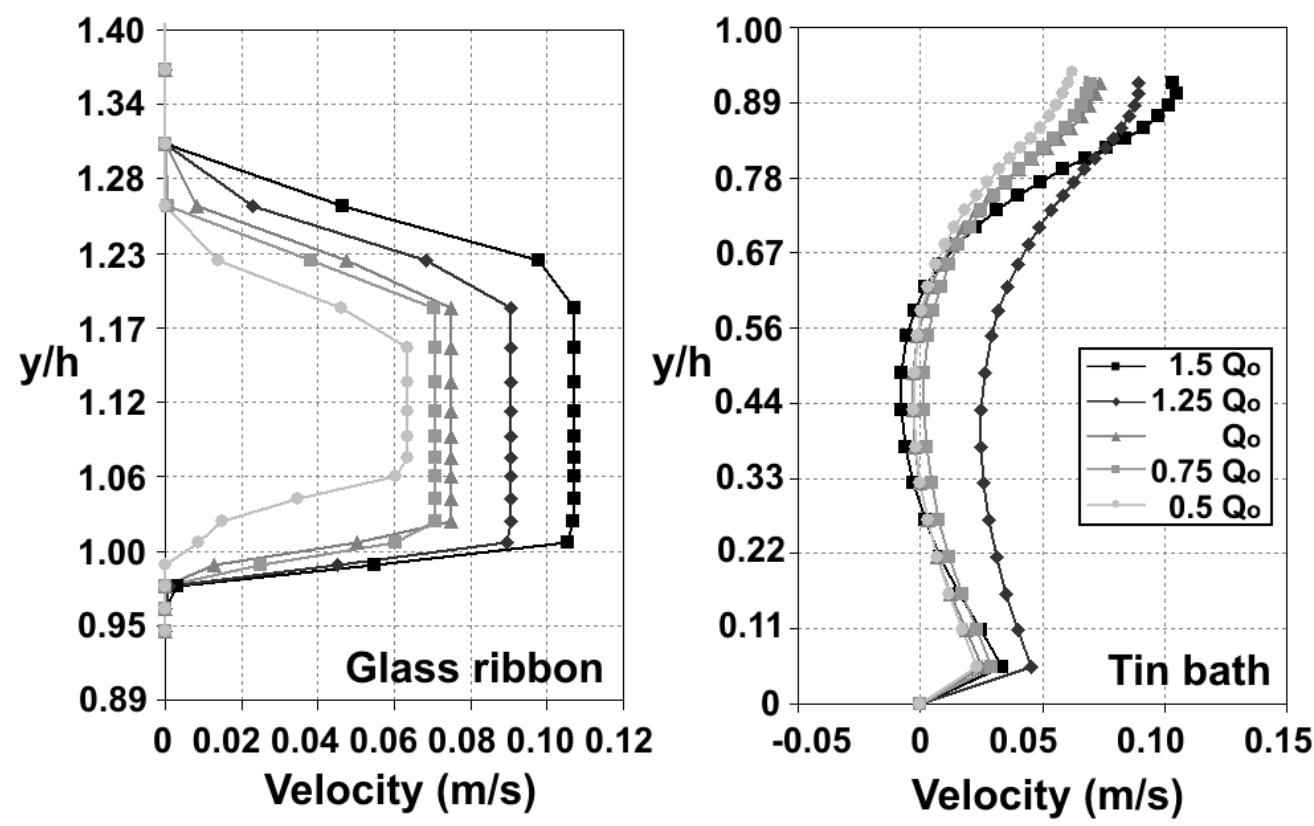

Fig. 18. Vertical distributions of glass and tin velocities in the central plane. 


\section{Other flow phenomena}

Both equilibrium thickness and wet back flow are important phenomena involved in the float fluid dynamics. Because of their extreme importance, the present simulation has been tested to determine if the numerical scheme captures these phenomena accurately. Since the thickness of the glass ribbon is not in equilibrium at the end zone of the restrictor tiles, it was necessary to enlarge the numerical domain to provide the correct evolution of the glass thickness.

Figure 19 shows a longitudinal view of the extended domain. The results for the nominal case have been represented here, though similar conclusions were obtained in other cases. A section placed approximately 3 meters downstream of the spout lip is observed in detail. As the glass ribbon flows downstream, the thickness is progressively reduced. In the meniscus area, a zoomed view of the wavefront demonstrates that the equilibrium thickness is effectively established there. The grid, superimposed on the ribbon in the representations, allows the estimation of the ribbon dimensions: $7-8 \mathrm{~mm}$. The denser vertical definition $(2 \mathrm{~mm}$ per cell) confirms that the meniscus is in hydrostatic equilibrium. On the contrary, the streamwise distribution of cells is too coarse. The mesh strategy followed in this case led to a progressive increment of the cell size when moving towards the domain exit. Thus, locations placed far way from the spout lip area present cells with an aspect ratio close to 10. As a consequence, the gradients of volume fraction are excessively sharp and the meniscus is described as a needle-shaped form, instead of the typical rounded radii. In any case, the model has proved accurate when capturing the value of the equilibrium thickness, in spite of the severe restrictions regarding mesh topologies. The extremely high computational effort that is needed to execute the numerical scheme prevented a finer spatial discretization.

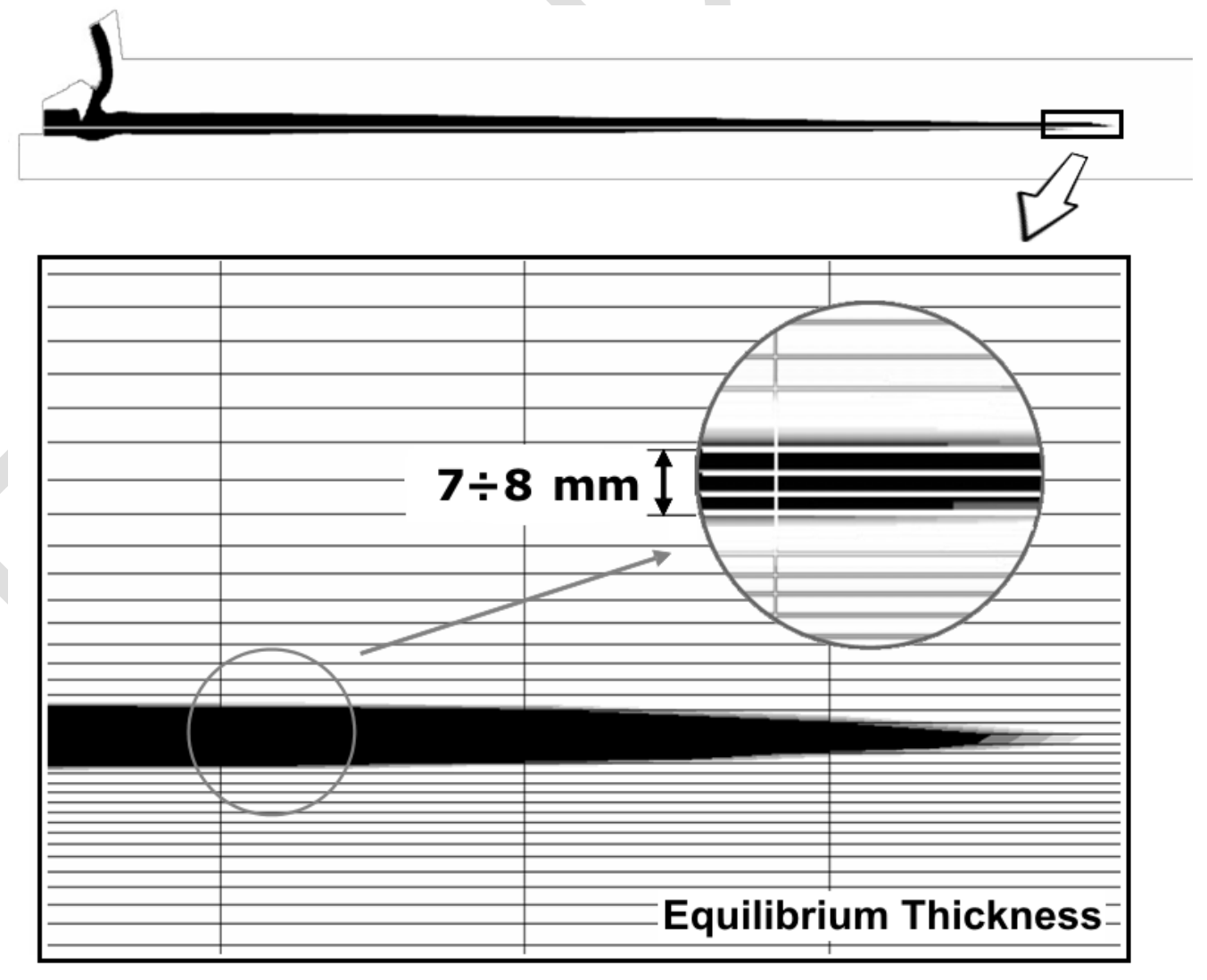

Fig. 19. Equilibrium Thickness described by the 3D model. 
In addition, the wet back flow phenomenon has also been described within the threedimensional case. Figure 20 shows a perspective of the glass ribbon formation beneath the spout lip for a larger mass flow rate $\left(1.5 \times Q_{0}\right)$. Both inner and outer interphases of the glass are represented, including the velocity fields. The reference magnitude of the velocity vectors is $10 \mathrm{~cm} / \mathrm{s}$. The impact of the pouring glass on the tin's surface creates a discontinuity in the glass ribbon, isolating the rear part of the ribbon and favouring the establishment of the wet back flow. This sharp discontinuity forces much of the glass to move downstream, as observed in the velocity vectors over the glass-tin interphase. The velocity distributions in the glass-air interphase are notably lower than in the glass-tin interphase, especially in the pool of glass accumulated next to the wet back tile. On the contrary, inner layers of glass are effectively deviated towards the casing, obliging them to flow with the main part of the glass in the direction of the float tank outlet. This is the phenomenon of the wet back flow, described in the literature and typically observed inside industrial floats.

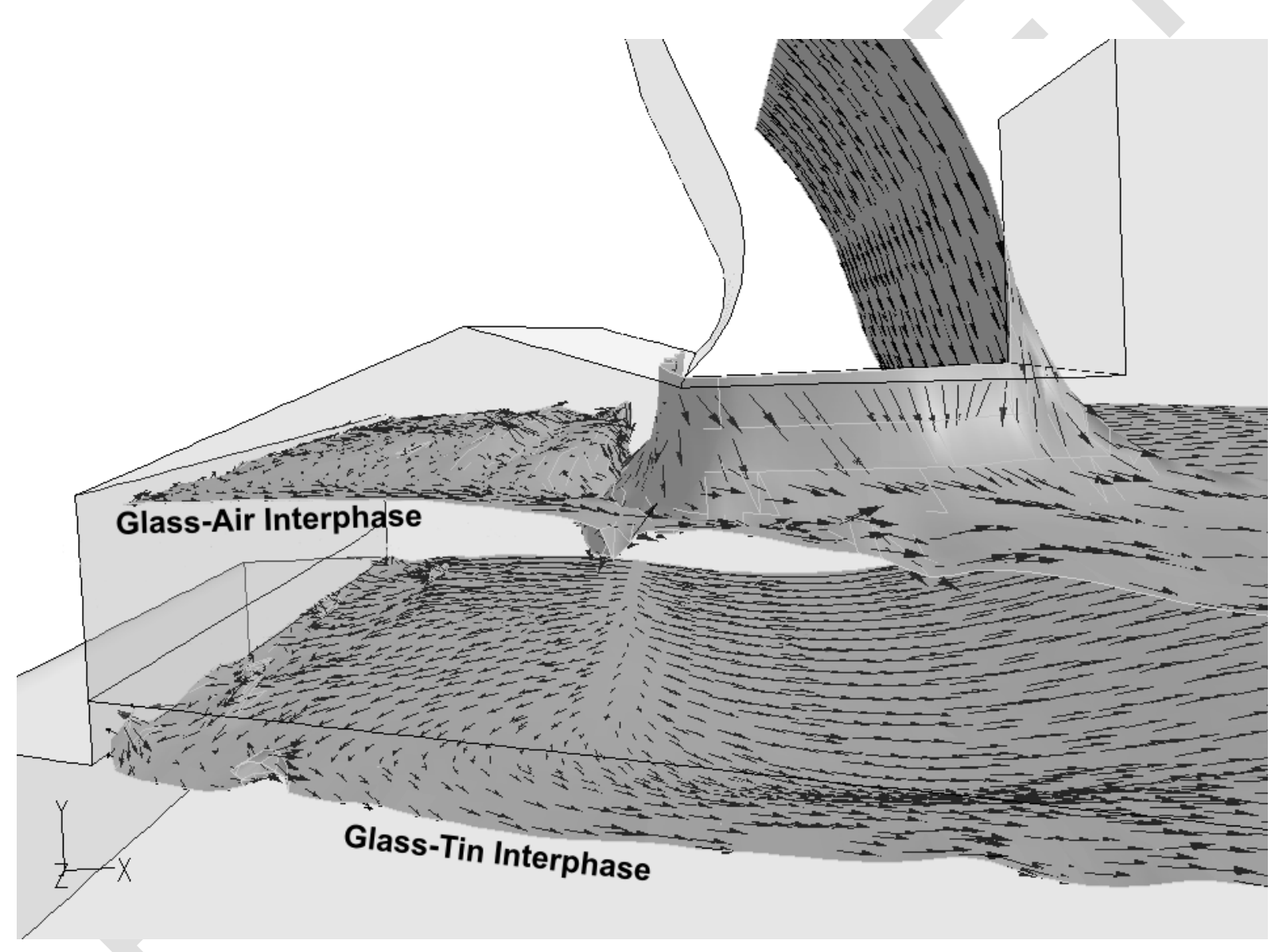

Fig. 20. Numerical results showing the wet back flow.

Finally, figure 21 shows a complementary effect that occurs due to the spreading mechanisms of the glass ribbon. First, the glass layers in contact with the refractory blocks of the spout lip became the ribbon outer layers after pouring onto the tin's surface. Note that the inclusion of abrasive particles and impurities released from the lip could compromise quality. Due to this inversion in the glass layers, the defects are preserved in the upper surface of the ribbon, avoiding their inclusion in the bulk of the glass ribbon. Moreover, the deviation of the path lines towards the lateral casing, as a result of the wet back flow, pushes the defects and/or impurities towards the edges of the glass sheet, so they can be easily removed later 
when cutting the marks of the top rollers. Figure 21 confirms this lateral displacement with a representation of the path lines released from the inlet.

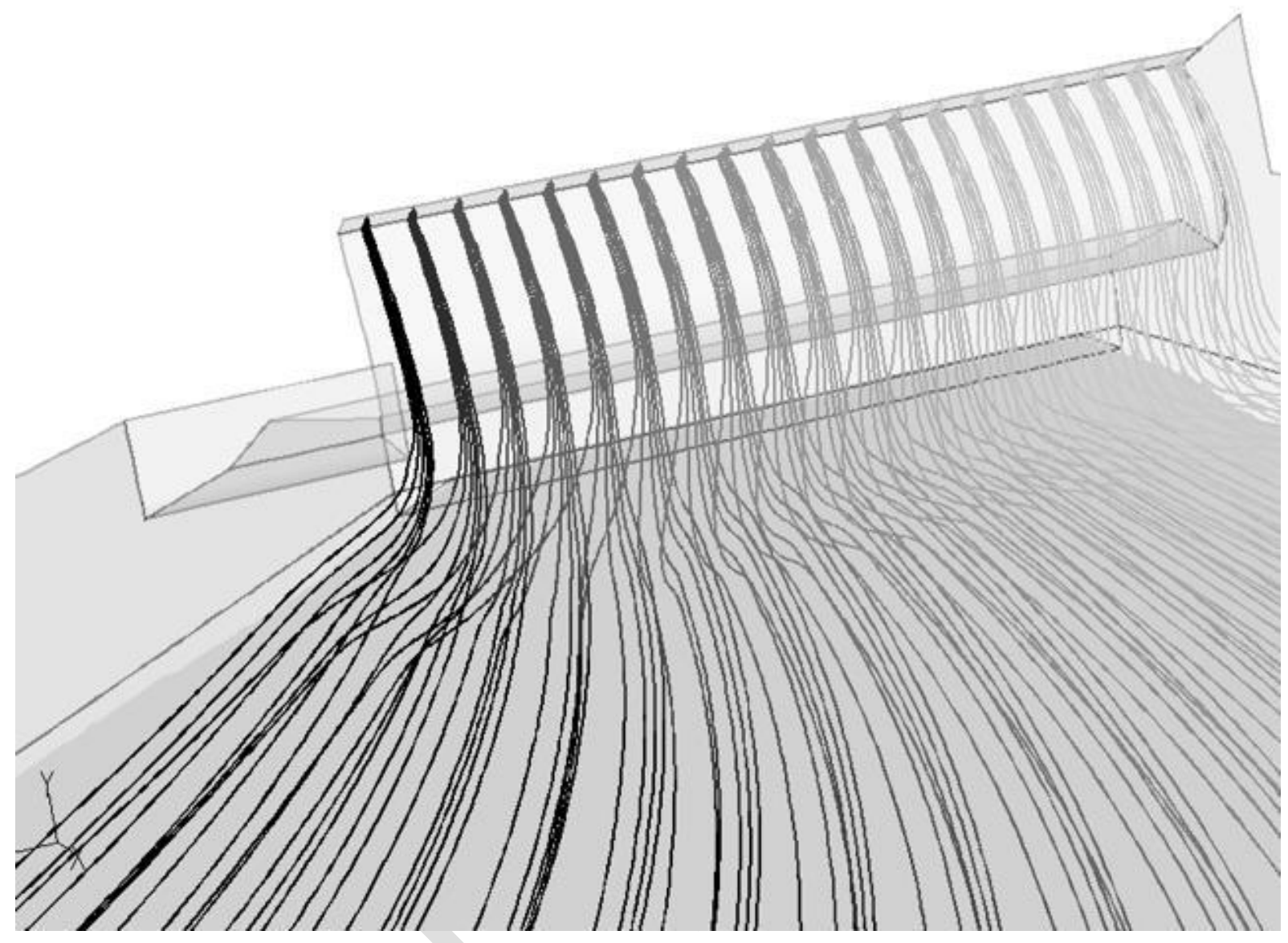

Fig. 21. Lateral displacement of path lines.

\section{CONCLUSION}

The present paper has presented a numerical methodology to analyze the pouring and spreading out mechanisms of molten glass on the tin bath in the flat glass forming process. The computational technique has required the employment of a three-phase modelling based on the VOF algorithm with CSF reformulation to address the transient tracking of the glass ribbon interphases.

Firstly, a complete analysis of the flat glass manufacturing process has been presented, regarding particular insight on the fluid dynamics of the tin bath entrance area. Following, a two-dimensional approach of the fluid transport was developed for validation purposes. Numerical and physical parameters were fixed in order to obtain an accurate description of the glass ribbon formation under conditions of numerical stability. Basic trends were established, satisfactorily approaching the experimental evidence inside real industrial floats. Also, different operating conditions of the float were tested, resulting in reasonable predictions of the glass ribbon conditions. The next step was the definition of a full 3D model to complete the analysis with a real geometry. Typical flow phenomena like the establishment of the equilibrium thickness or the formation of wet back flow were properly modelled in this case. The operating conditions have been seen to play an important role in the reinforcement of the wet back flow, especially at off-design performance of the float. On the contrary, the 
equilibrium thickness was shown to be an invariant parameter with the mass flow rate, as expected from theoretical considerations.

Further research must be focused on the improvement of the model, enhancing the numerical description and increasing the spatial discretizations. The introduction of rheological state equations for the glass phase combined with the resolution of thermal gradients would provide more valuable data, thus achieving a more complete validation of the simulations. On this point, not only the impact of the operating conditions on the float performance is of interest, but also the analysis of different glass viscosities would provide a more comprehensive approach to the fluid dynamics of the flat glass forming process.

\section{ACKNOWLEDGEMENTS}

The authors would like to thank the financial support provided by the Centre for Investigation and Development of Avilés (CIDA) under the leadership of Saint-Gobain Cristalería (Spain). Also the helpful suggestions of the technical staff of Saint-Gobain Recherche (SGR) are gratefully acknowledged.

\section{NOMENCLATURE}

$$
\begin{aligned}
& \mathrm{b}=\text { Unit width, [m] } \\
& \mathrm{BC}=\text { Boundary Condition } \\
& c, \mathbb{Q}=\text { Transition functions } \\
& \mathrm{Ca}=\text { Capillary number, }[-] \\
& \mathrm{CFD}=\text { Computational Fluid Dynamics } \\
& \mathrm{CFL}=\text { Courant-Friedrichs-Levy number } \\
& \mathrm{CSF}=\text { Continuum Surface Force } \\
& \mathrm{e}=\text { Glass film thickness, }[\mathrm{mm}] \\
& \mathrm{F}=\text { Specific volumetric force, }\left[\mathrm{N} / \mathrm{m}^{3}\right] \\
& \mathrm{g}=\text { Gravitational force, }\left[\mathrm{m}^{2} / \mathrm{s}\right] \\
& \mathrm{h}=\text { Initial tin level, [mm] } \\
& \mathrm{H}=\text { Height of lip nose, [mm] } \\
& \mathrm{k}=\text { Turbulent kinetic energy, }\left[\mathrm{m}^{2} / \mathrm{s}^{2}\right] \\
& \stackrel{\mathbf{l}}{n}=\text { Normal unit } \\
& \text { PISO }=\text { Pressure-Implicit with Splitting of Operators } \\
& \text { PLIC }=\text { Piecewise Linear Interface Calculation } \\
& Q, Q_{o}=\text { Flow rate and nominal flow rate, [ton/day] } \\
& \stackrel{r}{r}=(x, y, z)=\text { Position vector } \\
& \mathrm{Re}=\text { Reynolds number, [-] } \\
& \text { RSM }=\text { Reynolds Stress Model } \\
& \mathrm{RT}=\text { Restrictor Tile } \\
& \text { SLIC }=\text { Simple Line Interface Calculation } \\
& \mathrm{t}=\text { Time, }[\mathrm{s}] \\
& \mathrm{T}=\text { Equilibrium thickness, }[\mathrm{mm}] \\
& \stackrel{\mathrm{r}}{v}=(u, v, w)=\text { Velocity vectors } \\
& \text { VOF }=\text { Volume-of-fluid } \\
& \mathrm{We}=\text { Weber number, [-] } \\
& \text { WBT }=\text { Wet Back Tile }
\end{aligned}
$$


$\mathrm{X}, \mathrm{Y}, \mathrm{Z}=$ Cartesian coordinate system axes

\section{Greek Letters}

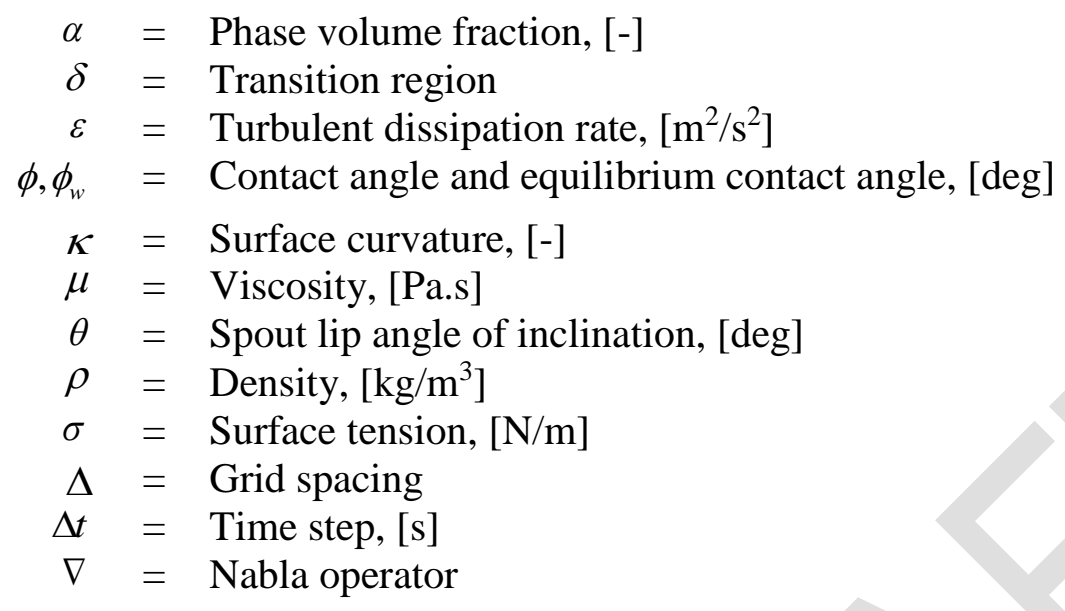

\section{Superscripts and Subscripts}

$$
\begin{aligned}
g & =\text { Glass } \\
t & =\text { Tin } \\
g t & =\text { Glass-Tin } \\
\text { lip } & =\text { Lip section } \\
q & =\text { Qth-phase } \\
i, j & =\text { Phases i \&j } \\
S & =\text { Surface } \\
V & =\text { Volumetric }
\end{aligned}
$$

\section{REFERENCES}

1. Pilkington LAB. The float glass process. Proc. Roy. Soc. London 1969; 314: 1-25.

2. Uhlmann DR, Kreidl NJ. Glass: Science and Technology, Vol. 2, Processing; I: 83-100. Academic Press, NY, 1984.

3. Hunt JCR. Industrial and Environmental Fluid Mechanics. Annual Review of Fluid Mechanics 1991; 23: 1-41.

4. Prieto M, Díaz J, Egusquiza E. Analysis of the fluid-dynamic and thermal behaviour of a tin bath in float glass manufacturing. International Journal of Thermal Sciences 2002; 41: 348-359.

5. Patankar SV. Computational modelling of flow and heat transfer in industrial applications. International Journal of Heat and Fluid Flow 2002; 23: 222-231.

6. Johansen ST. Multiphase flow modeling of metallurgical flows. Experimental, Thermal and Fluid Science 2002; 26: 739-745.

7. Kamihori T, Iga M, Kakihara S, Mase H. An integrated mathematical model of float process. Journal of Non-Crystalline Solids 1994; 177: 363-371.

8. Ban N, Kamihori T, Takamuku H. A study of the behavior of volatiles in the float process. Journal of Non-Crystalline Solids 2004; 345\&346: 777-781.

9. Narayanaswamy OS. A one dimensional model of stretching float glass. Journal of the American Ceramic Society 1977; 60(1-2): 1-5. 
10. Narayanaswamy OS. Computer simulation of float glass stretching. Journal of the American Ceramic Society 1981, 64(11): 666-670.

11. Schill P. Coupled method of tin bath and glass ribbon formation during float process. Proceedings of the $7^{\text {th }}$ International Conference on Advances in Fusion and Processing of Glass, 27-31 July, 2003, Rochester, NY, USA.

12. Pop SR. Modeling and simulation of the float glass process. Ph.D. Thesis 2005. University of Kaiserslautern, Germany.

13. Li G, Jinn JT, Wu WT, Oh SI, Recent developments and applications of threedimensional finite element modelling in bulk forming processes. Journal of Materials Processing Technology 2001; 113: 40-45.

14. Rienstra SW, Chandra TD, Analytical approximations to the viscous glass flow problem in the mould-plunger pressing process, including an investigation of boundary conditions. Journal of Engineering Mathematics 2001; 39(1): 241-259.

15. Murnane RA, Johnson WW, Moreland NJ. The analysis of glass melting processes using three-dimensional finite elements. International Journal for Numerical Methods in Fluids 1988; 8: 1491-1511.

16. Brown M. A review of research in numerical simulation for the glass-pressing process. Proceedings of the IMECHE Part B Journal of Engineering Manufacture 2007; 221: 1377-1386.

17. Wang G, Huang PG, Zhang YM. Numerical analysis of metal transfer in gas metal arc welding. Metallurgical and Material Transactions B 2003; 34(B): 345-353.

18. Fujimoto H, Shiotani Y, Tong AY, Hama T, Takuda H. Three-dimensional numerical analysis of the deformation behavior of droplets impinging onto a solid substrate. International Journal of Multiphase Flow 2007; 33: 317-332.

19. Mortensen D. A mathematical model of the heat and fluid flows in direct-chill casting of aluminium sheet ingots and billets. Metallurgical and Material Transactions B 1999; 30(B): 119-133.

20. Edge CK. Float Glass. Adv. Ceram. 1986; 18: 43-49.

21. Doyle PJ. Glass Making Today: 188-191. Ed. Portcullis Press Redhill, Great Britain, 1979.

22. Falleroni CA, Edge CK. Float glass technology: the bath atmosphere system. $P P G$ Technology Journal 1996; 2(15): 61-70.

23. Edge CK. Float glass manufacture: The control of tin flows. PPG Technology Journal 1995; 1(13): 31-42.

24. Bird RB, Stewart WE, Lightfroot EN, Transport Phenomena, $2^{\text {nd }}$. Ed. John Wiley \& Sons Inc, NY, New York.

25. Charnock H. Physics Bulletin 1970; 21: 153-156.

26. Cobo A. Estudio del equilibrio de flotación del vidrio sobre el estaño. Determinación del espesor de equilibrio. CIDA Library. Internal Communications. Saint-Gobain.

27. Hirt CW, Nichols BD. Volume of fluid (VOF) method for the dynamics of free boundaries. Journal of Computational Physics 1981; 39: 201-225.

28. Brackbill JU, Kothe DB, Zemach C. A continuum method for modeling surface tension. Journal of Computational Physics 1992; 100(2): 335-354.

29. Rider WJ, Kothe DB, Mosso SJ, Cerutti JH. Accurate solution algorithms for incompressible multiphase flows. AIAAJ 1995. AIAA95-0699.

30. Brackbill JU, Kothe DB. Dynamical modeling of surface tension. Los Alamos National Laboratory (LANL) Report 1996. LA-UR-96-1706.

31. Kothe DB, Rider WJ, Mosso SJ, Brock JS, Hochstein JI. Volume tracking of interfaces having surface tension in two and three dimensions". AIAAJ 1996. AIAA96-0859. 
32. Celic A, Zilliac G. Computational study of surface tension and wall adhesion effects on an oil film flow underneath an air boundary layer. NASA/TM-1998-112230.

33. Williams MW, Kothe DB, Puckett EG. Accuracy and convergence of continuum surface tension models. Los Alamos National Laboratory (LANL) Report 1998. LA-UR-98-2268.

34. FLUENT Inc. FLUENT® User`s Guide - Release 6.1, FLUENT Inc. 2003. Lebanon, $\mathrm{NH}$, USA.

35. Theodorakakos A, Bergeles G. Simulation of sharp gas-liquid interface using VOF method and adaptive grid local refinement around the interface. International Journal for Numerical Methods in Fluids 2004; 45: 421-439.

36. Mohapatra PK, Bhallamudi SM, Eswaran V. Numerical study of flows with multiple free surfaces. International Journal for Numerical Methods in Fluids 2001; 36: 165-184. 
This document is a pre-print version of the scientific paper published by Wiley. It has been released by the authors to fulfill all the publisher requirements established for Article Sharing:

https://authorservices. wiley.com/author-resources/Journal-

Authors/licensing/self-archiving.html

This is the pre-peer reviewed version of the article which has been published in final form at the International Journal of Numerical Methods in Fluids. This version may be used for non-commercial purposes in accordance with Wiley

Terms and Conditions for Use of Self-Archived Versions

\section{(2) $\odot \Theta \Theta$}

(C) 2019. This manuscript version is made available under the Creative Commons Attribution-NonCommercial-NoDerivatives 4.0 International License (CC-BY-NC-ND 4.0 license) http://creativecommons.org/licenses/by-nc-nd/4.0/ 